\title{
Bose-Fermi Chern-Simons dualities in the Higgsed phase
}

\author{
Sayantan Choudhury, ${ }^{a}$ Anshuman Dey, ${ }^{b}$ Indranil Halder, ${ }^{b}$ Sachin Jain, ${ }^{c}$ \\ Lavneet Janagal, ${ }^{b}$ Shiraz Minwalla ${ }^{b}$ and Naveen Prabhakar ${ }^{b}$ \\ ${ }^{a}$ Theoretical Cosmology Group, Max Planck Institute for Gravitational Physics, \\ Albert Einstein Institute, \\ Am Mühlenberg 1, 14476 Potsdam-Golm, Germany \\ ${ }^{b}$ Department of Theoretical Physics, Tata Institute of Fundamental Research, \\ Homi Bhabha Rd, Mumbai 400005, India \\ ${ }^{c}$ Indian Institute of Science Education and Research, \\ Homi Bhabha Rd, Pashan, Pune 411 008, India \\ E-mail: sayantan.choudhury@aei.mpg.de, anshuman@theory.tifr.res.in, \\ indranil.halder@tifr.res.in, sachin.jain@iiserpune.ac.in, \\ lavneet@theory.tifr.res.in, minwalla@theory.tifr.res.in, \\ naveensp@theory.tifr.res.in
}

ABSTRACT: It has been conjectured that fermions minimally coupled to a Chern-Simons gauge field define a conformal field theory (CFT) that is level-rank dual to ChernSimons gauged Wilson-Fisher Bosons. The CFTs in question admit relevant deformations parametrized by a real mass. When the mass deformation is positive, the duality of the two deformed theories has previously been checked in detail in the large $N$ limit by comparing explicit all orders results on both sides of the duality. In this paper we perform a similar check for the case of negative mass deformations. In this case the bosonic field condenses triggering the Higgs mechanism. The effective excitations in this phase are massive $W$ bosons. By summing all leading large $N$ graphs involving these $W$ bosons we find an all orders (in the 't Hooft coupling) result for the thermal free energy of the bosonic theory in the condensed phase. Our final answer perfectly matches the previously obtained fermionic free energy under the conjectured duality map.

Keywords: 1/N Expansion, Chern-Simons Theories, Duality in Gauge Field Theories

ArXiv EPrint: 1804.08635 


\section{Contents}

1 Introduction 1

1.1 Theories and conjectured dualities 3

1.2 Recap of known results 4

1.2.1 Structure of the large $N$ partition function 4

1.2.2 Free energies and gap equations 5

1.3 Computations in the Higgsed phase of the bosonic theory 8

2 Finite temperature partition function at large $N_{B} \quad 8$

2.1 The effective action in terms of $W$ bosons 8

2.2 Reducing the evaluation of the partition function to saddle point equations 11

$\begin{array}{lll}2.3 & \text { Dynamics in terms of singlet fields } & 15\end{array}$

2.4 A symmetry of the gap equations 16

2.5 Reduction to integral equations of a single variable 18

2.6 Dimensional regularization 22

2.7 Performing the angular integrals 22

2.8 Differential equations for the unknowns 23

2.9 Solving the gap equations 23

2.9.1 Determining $F_{4} \quad 23$

2.9.2 A subtlety in $F_{4} \quad 25$

$\begin{array}{ll}2.9 .3 \text { A curious observation } & 26\end{array}$

$\begin{array}{lll}2.9 .4 & \text { Determining } F_{2} & 27\end{array}$

2.9.5 Determination of the mass 28

2.9.6 Solving for $F_{3}$ and $F_{1} \quad 30$

2.9.7 Adding a chemical potential 31

3 The free energy 32

4 Discussion $\quad 37$

A Review of known results and a prediction for the Higgsed phase $\quad 39$

\section{Introduction}

It has recently been conjectured that fermions coupled to Chern-Simons gauge theories in certain representations of the gauge group are dual to bosons coupled, roughly speaking, to level-rank dual representations of the level-rank dual Chern-Simons gauge theories. The initial reason to suspect such a duality arose [1] from the study of conjectured dual bulk Vasiliev duals of these theories [1-5]. Moreover in the papers $[1,6,7]$ it was demonstrated 
that Chern-Simons theories with matter in the fundamental representation are 'solvable' in the large $N$ limit. Using the results of $[6,7]$ and the Schwinger-Dyson techniques developed in [1]), the authors of $[8,9]$ demonstrated that the three point functions of single trace operators on the two sides of the duality match at their conformal points provided the levels and ranks of these theories are exchanged under the duality ${ }^{1}$ and so provided the first concrete conjecture for the including a map between dual parameters [8]. ${ }^{2}$

While the matching of correlators between the two conformal theories suggests that they are dual, this evidence alone is less than clinching as the structure of large $N$ three point functions of single trace operators in these theories is highly constrained by approximate higher spin symmetries [6, 7]. Compelling additional evidence for these dualities - at least at large $N$ - comes from matching thermal partition functions [1, 16-25] and S-matrices [26-31]. It turns out that these quantities can both be explicitly computed at large $N$ independently in the bosonic and fermionic theories using the techniques first introduced in [1] and match perfectly between bosons and fermions under the same duality map proposed in [8], establishing the duality between these theories beyond reasonable doubt, at least at leading order in the large $N$ limit. The authors of $[22,24]$ were also able to construct pairs of dual [32] RG flows that originate at the $\mathcal{N}=2$ supersymmetric field theory that terminate in the IR at the critical boson and regular fermion theories respectively. The fact that the supersymmetric duality of [32] holds at finite $N$ supplies evidence for the validity of Bose-Fermi dualities at finite (if large) $N .^{3}$

Chern-Simons coupled regular fermion and critical boson theory CFTs each admit a massive deformation labelled by a real mass, that map to each other under duality. Turning on this mass deformation triggers an RG flow. Positive and negative mass deformations both lead to gapped theories or more precisely pure Chern-Simons topological field theories (TFT)s. The low energy TFTs are different for the two signs of mass. A change in sign of the fermion mass from positive to negative decreases the level of the effective low energy topological Chern-Simons theory by one unit. On the other hand, changing the boson mass changes from positive to negative is expected to cause the boson to condense and so to reduce the rank of the low energy topological Chern-Simons theory by one unit. These two effects map to each other under level-rank duality [8]. In the large $N$ limit there is already considerable direct calculational evidence for the duality between the two CFTs after a mass deformation for one sign of the mass, as we now briefly review. ${ }^{4}$

Correlation functions are much more constrained at fixed points than along RG flows. As a consequence there have been no exact results for correlation functions in the mass deformed bosonic and fermionic theories. It turns out, however, that the thermal partition functions and S-matrices of both theories are roughly as easy to compute at large $N$ in the mass deformed theories (for both signs of the fermionic mass and for positive bosonic mass)

\footnotetext{
${ }^{1}$ See $[10,11]$ for further results on correlation functions.

${ }^{2}$ See $[12-15]$ for more precise versions of the duality map.

${ }^{3}$ See $[5,9,10,16,17,20,23-25,33-46]$ for other large- $N$ computations that provide additional evidence for this duality.

${ }^{4}$ Of course a duality between two CFTs implies a duality between dual pairs of relevant deformations of these CFTs. So the matching of physical quantities after deformation can be regarded as strong additional evidence for the duality between the parent CFTs.
} 
as at the fixed point. Explicit all-orders results for the partition function and S-matrix are already available for positive bosonic masses and fermionic masses of both signs; and - to the extent that they can be compared - match perfectly under duality [1, 16-25]. However, explicit all-orders results for the negative mass deformed bosonic theory have not been as easy to obtain. At the calculational level a negative bosonic mass causes the bosons to condense, completely changing the nature of the mathematical problem to be solved. In order to determine the free energy and S-matrices of the bosonic theory in the condensed phase, in other words, one is required to solve a new mathematical problem that is not a small deformation of the analogous problem solved at the conformal fixed point.

In this paper we solve this 'new mathematical problem' for the bosonic free energy at finite temperature, and thereby present an all-orders 'solution' of the bosonic theory with a negative mass deformation. We proceed as follows. First we note that the effective excitations in the Higgsed bosonic phase are $W$ bosons. We work in a mixed unitary lightcone gauge that is convenient for our problem, and reduce the original scalar ChernSimons Lagrangian to a Lagrangian that describes the interaction of the charged $W$ bosons with the unbroken part of the gauge group. We then use Schwinger-Dyson methods to sum all diagrams that contribute at leading order in the large $N$ limit to the thermal propagator of the $\mathrm{W}$ bosons. The free energy is then obtained by sewing this exact thermal propagator on itself and adding in some appropriate counterterms. Our final result for the thermal free energy (and thermal mass of the $W$ bosons) turns out to match perfectly with the previously obtained dual fermionic results.

We leave the generalization of the computations of this paper to S-matrices to future work. We also work only with critical bosons, leaving the generalization to other theories to future work. We also work only in the strict large $N$ limit. See [12-14, 47-60] for exciting recent progress on the study of these dualities, and additional checks of the dualities, at finite $N$.

In the rest of this introduction we provide a more detailed description of the theories we study, our explicit results in the context of what was already known, as well as the interesting physical implications of our computations.

\subsection{Theories and conjectured dualities}

The two classes of theories we study in this paper are Regular Fermion (RF) theories defined by the Lagrangian

$$
S_{\mathrm{RF}}[\psi]=S_{\mathrm{CS}}+\int d^{3} x\left(\bar{\psi} \gamma_{\mu} D^{\mu} \psi+m_{F}^{\mathrm{reg}} \bar{\psi} \psi\right),
$$

and the so called Critical Boson (CB) theory defined by the Lagrangian

$$
S_{\mathrm{CB}}\left[\phi, \sigma_{B}\right]=S_{\mathrm{CS}}+\int d^{3} x\left[D_{\mu} \bar{\phi} D^{\mu} \phi+\sigma_{B}\left(\bar{\phi} \phi+\frac{N_{B}}{4 \pi} m_{B}^{\mathrm{cri}}\right)\right] .
$$

In the actions (1.1) and (1.2), $S_{\mathrm{CS}}$ denotes the pure gauge action for three dimensional gauge fields which is of the pure Chern-Simons form without admixture of a Yang-Mills 
term. ${ }^{5}$ The field $\sigma_{B}$ in (1.2) is a Lagrange multiplier field ${ }^{6}$ and its presence is the manifestation of the fact that we are studying the 'critical' or gauged Wilson-Fisher scalar theory rather than the regular or gauged free scalar theory.

In this paper we restrict our attention to the gauge groups $\mathrm{SU}\left(N_{F}\right)$ or $\mathrm{U}\left(N_{F}\right)$ (for the fermionic theory) and $\mathrm{SU}\left(N_{B}\right)$ or $\mathrm{U}\left(N_{B}\right)$ for the bosonic theory. The Chern-Simons level for the $\mathrm{SU}\left(N_{B}\right)$ Chern-Simons action in the bosonic theory will be denoted by the integer $k_{B}{ }^{7}$ We define levels of the fermionic theory to be the level of the pure ChernSimons theory obtained in the IR by giving the fermions a mass of the same sign as their level and integrating them out. With this definition, the rank of the $\mathrm{SU}\left(N_{F}\right)$ part of the fermionic theory is denoted by $k_{F} \cdot{ }^{8}$ In the large $N$ limit the $\mathrm{SU}(N)$ theories and the two $\mathrm{U}(N)$ theories all coincide, so we effectively treat them as identical and deal with them all together in the computations presented in this paper.

It has been conjectured that the fermionic and bosonic theories described above are dual to each other when their levels and ranks are related as follows

$$
k_{F}=-\operatorname{sgn}\left(k_{B}\right) N_{B}, \quad N_{F}=\left|k_{B}\right|
$$

In the large $N$ limit of these theories, instead of working with levels and ranks, it is useful to parametrize these theories by their 'renormalized' levels $\kappa_{F}, \kappa_{B}$ and 't Hooft couplings $\lambda_{F}, \lambda_{B}$ defined by

$$
\kappa_{B}=\operatorname{sgn}\left(k_{B}\right)\left(\left|k_{B}\right|+N_{B}\right), \quad \kappa_{F}=\operatorname{sgn}\left(k_{F}\right)\left(\left|k_{F}\right|+N_{F}\right), \quad \lambda_{F}=\frac{N_{F}}{\kappa_{F}}, \quad \lambda_{B}=\frac{N_{B}}{\kappa_{B}} .
$$

In terms of these variables, the conjectured duality map may be stated as

$$
\kappa_{F}=-\kappa_{B}, \quad \lambda_{F}=-\operatorname{sgn}\left(\lambda_{B}\right)+\lambda_{B} .
$$

At least in the large $N$ limit, the conjectured duality map for mass parameters between the bosonic and fermionic theories is given by

$$
m_{F}^{\mathrm{reg}}=-\lambda_{B} m_{B}^{\mathrm{cri}} .
$$

\subsection{Recap of known results}

\subsubsection{Structure of the large $N$ partition function}

It was demonstrated in [19] that the partition function of theories (1.1) and (1.2) on $S^{2} \times S^{1}$ can be evaluated (in a suitably coordinated high temperature and large $N$ limit) by following a two step procedure that we now outline.

\footnotetext{
${ }^{5}$ One way of giving precise meaning to the theories studied in this paper is by turning on a Yang Mill term with a small gauge coupling and then taking the limit in which this coupling goes to zero.

${ }^{6}$ One way of thinking of this field is as a Hubbard-Stratonovich field that accounts for a $\phi^{4}$ interaction between the bosons. In the limit that the $\phi^{4}$ coupling becomes very large this quadratic term for the Hubbard-Stratonovich field vanishes, turning it into a Lagrange multiplier.

${ }^{7}$ In the case that the bosonic theory is $\mathrm{U}\left(N_{B}\right)$, the rank of its $\mathrm{U}(1)$ part is either $N_{B} k_{B}$ - in the case of the so called type II theory or $N_{B} \operatorname{sgn}\left(k_{B}\right)\left(\left|k_{B}\right|+N_{B}\right)$ in the case of the so called type 1 theory. See [50] for a generalization of the type 1 and type II theories to a more general set of so called $\left(k, k^{\prime}\right)$ theories.

${ }^{8}$ Once again, the case that the fermionic theory is $\mathrm{U}\left(N_{F}\right)$, the rank of its $\mathrm{U}(1)$ part is either $N_{F} k_{F}$ - in the case of the so called type II theory or $N_{B} \operatorname{sgn}\left(k_{F}\right)\left(\left|k_{F}\right|+N_{F}\right)$ in the case of the so called type 1 theory.
} 
In the first step we are instructed study the theory in question on $\mathbb{R}^{2} \times S^{1}$. Up to gauge transformations, the zero mode of the holonomy $U$ of the gauge field around $S^{1}$ is completely specified by its eigenvalues $e^{i \theta_{j}}$ where $j=1 \ldots N$ and

$$
\theta_{j} \in(-\pi, \pi]
$$

In the large $N$ limit, all information about the eigenvalues of the holonomy matrix is conveniently packaged into an eigenvalue distribution function $\rho(\alpha)$ defined by

$$
\rho(\alpha)=\frac{1}{N} \sum_{i=1}^{N} \delta\left(\alpha-\theta_{i}\right)
$$

To complete the first step we are instructed to evaluate the path integral of our theory at fixed values of the holonomy zero mode $U$. This path integral defines the 'free energy functional' $v[U],{ }^{9}$ via the schematic equation

$$
e^{-\mathcal{V}_{2} T^{2} v[U]}=\int_{\mathbb{R}^{2} \times S^{1}}[d \phi] e^{-S[\phi, U]} .
$$

where $\mathcal{V}_{2}$ is the volume of two dimensional space and $T$ is the temperature. In order to complete the evaluation of the partition function of interest, we are instructed to evaluate the unitary matrix integral

$$
\mathcal{Z}_{S^{2} \times S^{1}}=\int[d U]_{\mathrm{CS}} e^{-\mathcal{V}_{2} T^{2} v[U]} .
$$

(This is the second step of our procedure). Here $[d U]_{\mathrm{CS}}$ is a Chern-Simons modified Haar measure over $\mathrm{U}(N)$ (see [19] for full details). In the large $N$ limit under study, (1.9) is most conveniently evaluated in the saddle point approximation.

In this paper we focus entirely on the first step of this programme, i.e. the evaluation of $v[U]$ (or more appropriately $v[\rho]$ ) defined in (1.8). In order to investigate the actual physics of our theories (as opposed to simply verifying duality) one would have to study the matrix integral (1.9); we leave this to future work.

\subsubsection{Free energies and gap equations}

We now review what is already known about the functionals $v_{F}$ and $v_{B}$ i.e. $v$ evaluated using (1.8) starting with the fermionic theory (1.1) and the bosonic theory (1.2) respectively.

By explicitly evaluating an infinite sum of loop diagrams of the fermionic/bosonic fields one obtains off-shell free energy functionals $v_{F}\left(\left|c_{F}\right|, \rho_{F}\right)$ and $v_{B}\left(\left|c_{B}\right|, \rho_{B}\right)$ for these two theories respectively. Note that each of these off shell free energies depend on an auxiliary variable (i.e. $\left|c_{F}\right|$ or $\left|c_{B}\right|$ ) in addition to the holonomy eigenvalue distribution functions. The onshell free energy functionals defined in (1.8) are functions only of the holonomy fields; and are obtained from their off-shell counterparts by extremizing w.r.t. $\left|c_{F}\right|$ and $\left|c_{B}\right|$ respectively. The extremum values of $\left|c_{F}\right|$ and $\left|c_{B}\right|$ are physically interpreted as thermal

\footnotetext{
${ }^{9}$ We alternately use the notation $v[\rho]$ to denote the dependence of $v$ on the holonomy eigenvalue distribution $\rho(\alpha)(1.7)$ as is appropriate in the large $N$ limit.
} 
pole masses in units of the temperature for the fermionic/bosonic theories respectively. In case the off-shell free energy admits more than one extremum, we are instructed to choose the extremum with the lowest value of the free energy. The explicit results for the fermionic and bosonic off-shell free energies are

$$
\begin{aligned}
v_{B}\left(\left|c_{B}\right|, \rho_{B}\right)= & \frac{N_{B}}{6 \pi}\left[\frac{3}{2} \hat{m}_{B}^{\mathrm{cri}} c_{B}^{2}-\frac{1}{2}\left(\hat{m}_{B}^{\mathrm{cri}}\right)^{3}-\left|c_{B}\right|^{3}+\right. \\
& \left.+3 \int_{-\pi}^{\pi} \rho_{B}(\alpha) d \alpha \int_{\left|c_{B}\right|}^{\infty} d y y\left(\log \left(1-e^{-y-i \alpha-\nu}\right)+\log \left(1-e^{-y+i \alpha+\nu}\right)\right)\right], \\
v_{F}\left(\left|c_{F}\right|, \rho_{F}\right)= & \frac{N_{F}}{6 \pi}\left[\left|c_{F}\right|^{3} \frac{\left(\lambda_{F}-\operatorname{sgn}\left(X_{F}\right)\right)}{\lambda_{F}}+\frac{3}{2 \lambda_{F}} \hat{m}_{F}^{\mathrm{reg}} c_{F}^{2}-\frac{\left(\hat{m}_{F}^{\mathrm{reg}}\right)^{3}}{2 \lambda_{F}\left(\lambda_{F}-\operatorname{sgn}\left(X_{F}\right)\right)^{2}}\right. \\
& \left.-3 \int_{-\pi}^{\pi} \rho_{F}(\alpha) d \alpha \int_{\left|c_{F}\right|}^{\infty} d y y\left(\log \left(1+e^{-y-i \alpha-\nu}\right)+\log \left(1+e^{-y+i \alpha+\nu}\right)\right)\right] .
\end{aligned}
$$

Here, $\hat{m}_{B}^{\text {cri }}$ and $\hat{m}_{F}^{\text {reg }}$ are the masses divided by the temperature, and $\nu=\mu / T$ where $\mu$ is a chemical potential that couples to a charge under which all fundamental fields have unit positive charge while all antifundamental fields have unit negative charge. The quantity $X_{F}$ (and an analogous quantity $X_{B}$ that we will need in a moment) that appears in (1.10) are defined by

$$
\begin{aligned}
& X_{F}=2 \lambda_{F} \mathcal{C}+\hat{m}_{F}^{\text {reg }}, \\
& X_{B}=2 \lambda_{B} \mathcal{S}-\lambda_{B} \hat{m}_{B}^{\text {cri }}-\operatorname{sgn}\left(\lambda_{B}\right) \max \left(\left|c_{B}\right|,|\nu|\right),
\end{aligned}
$$

where

$$
\begin{aligned}
& \mathcal{C}\left(\left|c_{F}\right|, \nu\right)=\frac{1}{2} \int d \alpha \rho_{F}(\alpha)\left(\log \left(2 \cosh \left(\frac{\left|c_{F}\right|+i \alpha+\nu}{2}\right)\right)+\log \left(2 \cosh \left(\frac{\left|c_{F}\right|-i \alpha-\nu}{2}\right)\right)\right), \\
& \mathcal{S}\left(\left|c_{B}\right|, \nu\right)=\frac{1}{2} \int d \alpha \rho_{B}(\alpha)\left(\log \left(2 \sinh \left(\frac{\left|c_{B}\right|+i \alpha+\nu}{2}\right)\right)+\log \left(2 \sinh \left(\frac{\left|c_{B}\right|-i \alpha-\nu}{2}\right)\right)\right) .
\end{aligned}
$$

The expression for $v_{F}$ listed in (1.10) is expected to be complete. However the expression for $v_{B}$ was obtained by working about an unHiggsed bosonic vacuum. As we have explained earlier in this introduction, under certain circumstances - roughly for negative bosonic mass - we expect this to be the wrong vacuum for the bosonic theory. As a consequence we expect the expression for $v_{B}$ above to be correct only in a certain parametric regime. In this paper we will calculate the bosonic free energy in the complementary parametric regime. ${ }^{10}$

The condition that $v_{F}$ and $v_{B}$ are extremized on-shell yields an equation - called a gap equation - that can be used to determine $c_{F}$ and $c_{B}$ on-shell. The gap equations for the fermionic theory is

$$
\left|c_{F}\right|=\operatorname{sgn}\left(X_{F}\right)\left(2 \lambda_{F} \mathcal{C}\left(\left|c_{F}\right|, \nu\right)+\hat{m}_{F}^{\mathrm{reg}}\right)=\left|X_{F}\right|,
$$

\footnotetext{
${ }^{10}$ Our result for the free energy (1.10) is given in terms of the $\operatorname{logarithmic}$ function $\log (z)$. Here and through the rest of this paper this function is defined so that it is real when $z$ is real and positive, and so that it has a branch cut on the negative real axis in the $z$ plane.
} 
while the gap equation obtained by extremizing $v_{B}$ is

$$
2 \mathcal{S}\left(\left|c_{B}\right|, \nu\right)=\hat{m}_{B}^{\text {cri }}
$$

In appendix A below we review some properties of the gap equation. In particular we demonstrate that the bosonic gap equation (1.14) only has solutions provided

$$
-\operatorname{sgn}\left(\lambda_{B}\right) \operatorname{sgn}\left(X_{B}\right) \geq 0
$$

${ }^{11}$ When transformed to fermionic variables, (1.15) turns into the condition

$$
\operatorname{sgn}\left(\lambda_{F}\right) \operatorname{sgn}\left(X_{F}\right) \geq 0
$$

It turns out (see appendix A) that there is a one to one map between all solutions to the bosonic gap equation (1.14) and and those solutions to the fermionic gap equation (1.13) that obey (1.16).

However there also exist solutions to the fermionic gap equation that obey the complement of (1.16). The bosonic duals of these solutions have not been understood in the existing literature. However the duality map (see appendix A for details) can be used to recast the existing fermionic results into bosonic variables, yielding the gap equation

$$
2\left|c_{B}\right|=\left(2\left|\lambda_{B}\right| \tilde{\mathcal{S}}-\left|\lambda_{B}\right| \hat{m}_{B}^{\text {cri }}\right) .
$$

where

$$
\tilde{\mathcal{S}}=\left\{\begin{array}{ccc}
\mathcal{S} & \text { when } & |\nu|<\left|c_{B}\right| \\
\mathcal{S}-\frac{1}{2\left|\lambda_{B}\right|}\left(|\nu|-\left|c_{B}\right|\right) & \text { when } & |\nu|>\left|c_{B}\right|
\end{array} .\right.
$$

In this range of parameters the off-shell fermionic free energy can also be recast into bosonic language; we find

$$
\begin{aligned}
v_{B}\left(\left|c_{B}\right|, \rho_{B}\right)= & \frac{N_{B}}{6 \pi}\left[-\frac{|\hat{m}|^{3}}{\left|\lambda_{B}\right|}+3\left|\lambda_{B}\right||\hat{m}| \mathcal{S}^{2}+2\left|\lambda_{B}\right|^{2} \mathcal{S}^{3}\right. \\
& \left.-\left|c_{B}\right|^{3}+3 \int_{-\pi}^{\pi} \rho_{B}(\alpha) d \alpha \int_{\left|c_{B}\right|}^{\infty} d y y\left(\log \left(1+e^{-y-i \alpha-\nu}\right)+\log \left(1+e^{-y+i \alpha+\nu}\right)\right)\right],
\end{aligned}
$$

The equations (1.17) and (1.19) may be thought of as the predictions of duality for the gap equation and free energy of the bosonic theory in the condensed phase, i.e. when (1.15) is not obeyed. In this paper we will reproduce both these results from a direct evaluation of the bosonic path integral in the condensed phase. The exact agreement under duality of the bosonic and fermionic results may be thought of as a detailed new check of the duality conjecture.

\footnotetext{
${ }^{11}$ Eq. (1.15) should be thought of as effectively describing the parametric regime in which the bosonic free energy listed in (1.10) is valid. Outside this regime the free energy expression is obtained by the computation in the Higgsed phase that we perform in this paper.
} 


\subsection{Computations in the Higgsed phase of the bosonic theory}

Consider the theory (1.1) when $m_{B}^{\text {cri }}<0$. In this situation the equation of motion for the field $\sigma_{B}$ forces the modulus of $\bar{\phi} \phi$ to take a fixed nonzero constant value determined by $m_{B}^{\text {cri }}$. It is useful to work in the unitary gauge. This choice of gauge rotates $\phi$ to be real and to point in the $N_{B}$-th flavour direction in $\mathrm{SU}\left(N_{B}\right)$ colour space. ${ }^{12}$ As $|\phi|$ is a fixed constant, it follows that with this choice of gauge there are no degrees of freedom in $\phi$.

The 'condensation' of $\phi$ breaks $\mathrm{SU}\left(N_{B}\right)$ to $\mathrm{SU}\left(N_{B}-1\right)$. The gauge bosons of $\mathrm{SU}\left(N_{B}\right)$ split up into Chern-Simons coupled $\mathrm{SU}\left(N_{B}-1\right)$ gauge bosons, $N_{B}-1$ complex massive $W$ bosons that transform in the fundamental of $\mathrm{SU}\left(N_{B}-1\right)$ and a single real massive $Z$ boson that is uncharged under $\mathrm{SU}\left(N_{B}-1\right)$. The action that governs the interactions of these fields is easily worked out and is presented in (2.21), (2.22) below.

As in previous work (see [1] and several subsequent papers) we choose to adopt the lightcone gauge for the unbroken $\mathrm{SU}\left(N_{B}-1\right)$ gauge symmetry. Once we make this choice the Lagrangian is quadratic both in the $\mathrm{SU}\left(N_{B}-1\right)$ gauge fields and in the $Z$ bosons. It is thus possible to integrate both these fields out. This process generates a non-local quartic interaction between the $W$ bosons. The resultant effective theory usually is called a vector model in the literature on large $N$ models. The $W$ fields are $\mathrm{SU}\left(N_{B}-1\right)$ vectors with a non-local $W^{4}$ interaction. At this point we use standard large $N$ techniques to reduce the finite temperature path integral over $W$, in the large $N$ limit, to a set of nonlinear integral equations for the self-energy matrix $\Sigma^{\mu \nu}$ of the $W_{\mu}$ fields.

Using symmetries and other structural properties of the integral equations, it is possible to parametrize the four nonzero components of $\Sigma^{\mu \nu}$ by four unknown functions $F_{1}, \ldots, F_{4}$ of a single variable (see (2.53)). The integral equations for $\Sigma^{\mu \nu}$ reduce to a set of four nonlinear coupled integral equations for $F_{1}, \ldots, F_{4}$ (see (2.58)). Quite remarkably it turns out to be possible to solve these equations exactly, in terms of a single real constant $M$. $M$ is the thermal mass of the $W$ bosons and it, in turn, is required to obey a gap equation (2.101) which perfectly reproduces the prediction from duality (1.17), at least when $\left|c_{B}\right|>|\nu|$.

Next we plug our solution for $\Sigma^{\mu \nu}$ back into our large $N$ expression for the partition function. On-shell, we find that the final result for the free energy $v_{B}$ perfectly matches the prediction (1.19).

\section{Finite temperature partition function at large $N_{B}$}

\subsection{The effective action in terms of $W$ bosons}

Consider the mass deformed $\mathrm{SU}\left(N_{B}\right)$ critical boson theory defined by the Euclidean action $S_{\mathrm{E}},{ }^{13}$

$$
\begin{aligned}
S_{\mathrm{E}}=S_{\mathrm{CS}}+S_{\mathrm{B}}, \quad S_{\mathrm{CS}} & =\int d^{3} x i \epsilon^{\mu \nu \rho} \frac{\kappa_{B}}{4 \pi} \operatorname{Tr}\left(X_{\mu} \partial_{\nu} X_{\rho}-\frac{2 i}{3} X_{\mu} X_{\nu} X_{\rho}\right), \\
S_{\mathrm{B}} & =\int d^{3} x \sqrt{\operatorname{det} g}\left(D_{\mu} \bar{\phi} D^{\mu} \phi+\sigma_{B}\left(\bar{\phi} \phi+\frac{N_{B}}{4 \pi} m_{B}^{\mathrm{cri}}\right)\right) .
\end{aligned}
$$

\footnotetext{
${ }^{12}$ For definiteness sake, we work with the gauge group being $\mathrm{SU}\left(N_{B}\right)$ as opposed to $\mathrm{U}\left(N_{B}\right)$.

${ }^{13} \mathrm{We}$ follow the standard convention that the Euclidean partition function is given in terms of the Euclidean action by the path integral $\int[d \phi] e^{-S_{\mathrm{E}}[\phi]}$.
} 
where $D_{\mu} \phi=\partial_{\mu} \phi-i X_{\mu} \phi$. The fields $X_{\mu}$ are $N \times N$ hermitian matrices. Throughout this paper the gauge group generators are normalised such that $\operatorname{Tr}\left(T_{A} T_{B}\right)=\frac{1}{2} \delta_{A B}$. All through this paper we work with the dimensional regulation scheme (see subsection 2.6 below). With this choice of regulator the constant $\kappa_{B}$ that appears in (2.1) matches $\kappa_{B}$ defined in (1.4). In other words $\kappa_{B}$ may be identified with the 'renormalized' Chern-Simons level (see the discussion above (1.4)). We will present most of our formulae in terms of the 't Hooft coupling defined by $\lambda_{B}=\frac{N_{B}}{\kappa_{B}}$ (see (1.4)). Note that, by definition, $\left|\lambda_{B}\right| \leq 1$.

The field $\sigma_{B}$ plays the role of a Lagrange multiplier in the action (2.1). The $\sigma_{B}$ equation of motion

$$
\bar{\phi} \phi=-\frac{N_{B}}{4 \pi} m_{B}^{\mathrm{cri}},
$$

has no real solution when $m_{B}^{\text {cri }}$ is positive. In this 'standard' case one proceeds to analyse the theory assuming that the scalar field has a vacuum at $\phi=0$ and tests the self consistency of this assumption by demonstrating that the quantum effective action for $\sigma_{B}$ - evaluated by integrating out the $\phi$ fields — has a stable minimum.

In this paper we are interested in regime in which $m_{B}^{\text {cri }}$ is negative. In this case the situation is more straightforward. Let us define the real quantity $v$ by the equation

$$
\left|\kappa_{B}\right| v^{2}=-\frac{N_{B}}{4 \pi} m_{B}^{\mathrm{cri}} \Longrightarrow v^{2}=-\frac{\left|\lambda_{B}\right|}{4 \pi} m_{B}^{\mathrm{cri}}
$$

a definition that is sensible precisely because $m_{B}^{\text {cri }}$ is negative. The equation (2.2) may be rewritten as

$$
\bar{\phi} \phi=\left|\kappa_{B}\right| v^{2} .
$$

Clearly (2.2) now admits classical solutions, but the solution to this equation is not unique; given any solution one can always generate a new solution by performing a spacetime dependent $\mathrm{SU}\left(N_{B}\right)$ rotation of the original solution. Of course these rotations are simply gauge transformations, the solution to (2.2) can be made unique by making an appropriate choice of gauge. We adopt the so called 'unitary gauge' in which the fundamental field $\phi$ is always rotated to be real and to point in the $N_{B}$-th direction in colour space. The equation of motion (2.2) then determines the magnitude of $\phi$ at each point in spacetime and we find

$$
\phi^{i}=\delta^{i N_{B}} v \sqrt{\left|\kappa_{B}\right|}=\delta^{i N_{B}} \sqrt{\frac{N_{B}}{4 \pi}\left|m_{B}^{\mathrm{cri}}\right|} .
$$

With this choice the $\phi$ field is completely determined and effectively non dynamical, and the original $\mathrm{SU}\left(N_{B}\right)$ gauge symmetry is Higgsed down to $\mathrm{SU}\left(N_{B}-1\right)$.

Matter Chern-Simons theories are often thought of as theories which govern interaction of dynamical matter fields with non-dynamical gauge fields. In the current situation our gauge choice has frozen the matter field completely. So where has its degrees of freedom gone? Of course the answer to this question is familiar; symmetry breaking of the gauge group transfers the degrees of freedom of the matter field into the gauge field. More precisely, those gauge bosons that originally were in the adjoint of $\mathrm{SU}\left(N_{B}\right)$ - but are not in the adjoint of the residual $\mathrm{SU}\left(N_{B}-1\right)$, inherit the matter degrees of freedom and turn 
into propagating massive $W$ bosons after symmetry breaking. We now explain in detail how this works.

Let $\left(X_{\mu}\right)_{j}^{i}$ represent the $i j^{\text {th }}$ element of the matrix valued field $X_{\mu}$ in (2.1). The indices $i$ and $j$ run over the range $i, j=1, \ldots, N_{B}$. It is useful to separate out $i=N_{B}$ as special (same for $j$ ). Let $a, b$ denote indices that run from $1, \ldots, N_{B}-1$. Then we define

$$
\left(X_{\mu}\right)_{N_{B}}^{a}=\frac{W_{\mu}^{a}}{\sqrt{\kappa_{B}}}, \quad\left(X_{\mu}\right)_{b}^{N_{B}}=\frac{\left(\bar{W}_{\mu}\right)_{b}}{\sqrt{\kappa_{B}}}, \quad\left(X_{\mu}\right)_{N_{B}}^{N_{B}}=Z_{\mu}, \quad\left(X_{\mu}\right)_{b}^{a}=\left(A_{\mu}\right)_{b}^{a}-\frac{Z_{\mu}}{N_{B}-1} \delta_{b}^{a}
$$

where the traceless matrices $\left(A_{\mu}\right)_{b}^{a}$ are the gauge bosons of the unbroken $\mathrm{SU}\left(N_{B}-1\right)$ gauge group and $\left(W_{\mu}^{a}\right)^{*}=\left(\bar{W}_{\mu}\right)_{a}$, i.e. the fields $W_{\mu}$ and $\bar{W}_{\mu}$ are complex conjugates of each other. Notice that $W_{\mu}$ transforms in the fundamental while $\bar{W}_{\mu}$ transforms in the antifundamental of the unbroken gauge group $\mathrm{SU}\left(N_{B}-1\right)$.

Working in unitary gauge and with the decomposition described in (2.6) we obtain the Euclidean action

$$
\begin{aligned}
S_{\mathrm{E}}[A, W, Z]= & \frac{i \kappa_{B}}{4 \pi} \int \operatorname{Tr}\left(A d A-\frac{2 i}{3} A A A\right)+\frac{i}{4 \pi} \int\left[2 \bar{W} D W+\kappa_{B} Z d Z-2 i Z \bar{W} W\right] \\
& +\operatorname{sgn}\left(\kappa_{B}\right) v^{2} \int d^{3} x \sqrt{\operatorname{det} g}\left(\kappa_{B} Z_{\mu} Z^{\mu}+\bar{W}_{\mu} W^{\mu}\right)
\end{aligned}
$$

where $D_{\mu}=\partial_{\mu}-i A_{\mu}$ and the exterior product $A B C$ means $d^{3} x \epsilon^{\mu \nu \rho} A_{\mu} B_{\nu} C_{\rho}$.

At the linearized order the equation of motion for the field $A_{\mu}$ is simply $F_{\mu \nu}=0$, reflecting the fact that the field $A_{\mu}$ has no propagating degrees of freedom. On the other hand the linearized equations of motion for the fields $W_{\mu}$ and $Z_{\mu}$ are

$$
\frac{i \epsilon^{\mu \nu \rho}}{4 \pi} 2 \partial_{\nu} W_{\rho}+\operatorname{sgn}\left(\kappa_{B}\right) v^{2} W^{\mu}=0, \quad \frac{i \epsilon^{\mu \nu \rho}}{4 \pi} 2 \partial_{\nu} Z_{\rho}+\operatorname{sgn}\left(\kappa_{B}\right) v^{2} 2 Z^{\mu}=0
$$

It follows immediately from the divergence of (2.8) that $\partial_{\mu} W^{\mu}=\partial_{\mu} Z^{\mu}=0$. The equations (2.8) are easily solved in momentum space. Let us define

$$
W_{\mu}^{a}(x)=\int \frac{d^{3} q}{(2 \pi)^{3}} e^{i x \cdot q} W_{\mu}^{a}(q) .
$$

Then, (2.8) turns into

$$
\left(-\frac{\epsilon^{\mu \nu \rho} q_{\nu}}{2 \pi}+\operatorname{sgn}\left(\kappa_{B}\right) v^{2} g^{\mu \rho}\right) W_{\rho}^{a}(q)=0
$$

The equation (2.10) has solutions only when the matrix on the l.h.s. of (2.10) has a zero eigenvalue. This, in turn, is the case only when

$$
-g^{\mu \nu} q_{\mu} q_{\nu}=\left(2 \pi v^{2}\right)^{2} \equiv m_{W}^{2}
$$

It follows that the $W$-boson $W_{\mu}$ is a propagating field with mass $m_{W}=+2 \pi v^{2}$. For every $q_{\mu}$ (at fixed $v^{2}$ ) for which (2.11) is obeyed, the solution to (2.10) is uniquely determined up to a single complex number (either $W_{+}$or $W_{-}$for example). It follows, in other words, that the fields $W_{\mu}^{a}$ have the same number of degrees of freedom as a standard massive 
scalar field of mass $m_{W}$. In a similar way the $Z$ boson $Z_{\mu}$ has as as many solutions as a single real scalar field of mass $2 m_{W}$. The total number of degrees of freedom of the $W, Z$ system is thus that of $2\left(N_{B}-1\right)+1=2 N_{B}-1$ real massive scalars. This is precisely the number of degrees of freedom in the scalar field once its modulus has been frozen by the $\sigma_{B}$ equation of motion. The condensation of $\phi$ simply 'transmutes' these degrees of freedom from a spin zero scalar to a spin \pm 1 vector. ${ }^{14}$

\subsection{Reducing the evaluation of the partition function to saddle point equations}

In the rest of this section we will evaluate the finite temperature partition function

$$
\mathcal{Z}=\int[d A d W d Z] e^{-S_{\mathrm{E}}[A, W, Z]},
$$

where the action $S_{\mathrm{E}}$ was defined in (2.7) and the path integral is evaluated over the Euclidean manifold $\mathbb{R}^{2} \times S^{1}$.

Adapting the explanation of [19] to the current context, it is possible to convince oneself that the path integral (2.12) may be evaluated (in the coordinated high temperature and large $N_{B}$ limit described in [19]) by following a two step process. The first step in this process is to evaluate the path integral over the fields of $(2.7)$ on $\mathbb{R}^{2} \times S^{1}$ at fixed values of the holonomy fields of the unbroken gauge group $\mathrm{SU}\left(N_{B}-1\right)$. The second step in this process is to regard the result of the first path integral as an effective action for the holonomy matrix, and then use this action to perform an integral over the holonomy matrices. Both steps in this process can be practically carried through in the large $N_{B}$ limit (the second step involves solving a saddle point equation for the holonomies). In this section we concentrate entirely on the first step, leaving the second step for later work.

In order to proceed with our computation we need to fix the unbroken $\mathrm{SU}\left(N_{B}-1\right)$ gauge invariance in the action (2.7). Following [1] (and more or less every other subsequent successful large $N$ computation in matter Chern-Simons theories) we work in the lightcone gauge $A_{-}=0 .{ }^{15}$ Once we adopt this gauge, the cubic term for $A$ vanishes in the ChernSimons action. The action (2.7) simplifies to

$$
\begin{aligned}
S_{\mathrm{E}}[A, W, Z]= & \frac{i}{4 \pi} \int d^{3} x \operatorname{Tr}\left(\kappa_{B} \epsilon^{\tilde{\mu}-\tilde{\nu}} A_{\tilde{\mu}} \partial_{-} A_{\tilde{\nu}}\right)+\int d^{3} x \bar{W}_{\mu}\left(\frac{i}{2 \pi} \epsilon^{\mu \nu \rho} \partial_{\nu}+\operatorname{sgn}\left(\kappa_{B}\right) v^{2} g^{\mu \rho}\right) W_{\rho} \\
& +\int d^{3} x\left(Z_{\mu}\left(\frac{i \kappa_{B}}{4 \pi} \epsilon^{\mu \nu \rho} \partial_{\nu}+g^{\mu \rho}\left|\kappa_{B}\right| v^{2}\right) Z_{\rho}\right)+\frac{1}{2 \pi} \int d^{3} x \epsilon^{\mu \nu \rho} \bar{W}_{\rho}\left(A_{\mu}-Z_{\mu}\right) W_{\nu}
\end{aligned}
$$

\footnotetext{
${ }^{14}$ If we take the momentum of the $W$ boson to be in the 3 direction, it is easy to check that its polarization is in the $z=x+i y$ direction when $\kappa_{B}$ is positive but in the $\bar{z}=x-i y$ direction when $\kappa_{B}$ is negative (this follows from (2.10) using (2.11)). In other words the little group spin of our on-shell $W$ bosons equals $\operatorname{sgn}\left(\kappa_{B}\right)$.

${ }^{15}$ Our conventions are defined by

$$
x^{ \pm}=\frac{x^{1} \pm i x^{2}}{\sqrt{2}}, p_{\mp}=\frac{p^{1} \pm i p^{2}}{\sqrt{2}}, A_{\mp}=\frac{A^{1} \pm i A^{2}}{\sqrt{2}} .
$$

In our conventions, the nonzero components of the metric in lightcone coordinates are $g_{+-}=g_{-+}=g_{33}=1$, the Levi-Civita symbol is given by $\epsilon^{+-3}=\epsilon_{-+3}=-i$, and the Kronecker delta is given by $\delta_{\nu}^{\mu}=1$ if $\mu=\nu$ and 0 otherwise.
} 
where the indices $\tilde{\mu}, \ldots$ run over,+ 3 only. The first line of the action (2.14) contains the quadratic kinetic terms for the $\mathrm{SU}\left(N_{B}-1\right)$ gauge fields $A_{+}$and $A_{3} .{ }^{16}$ The second line of (2.14) has the kinetic term for the $\mathrm{SU}\left(N_{B}-1\right)$ fundamental field $W_{\mu}$ and its (antifundamental) complex conjugate $\bar{W}_{\mu}$. All gauge indices (which have been suppressed for readability) in (2.14) are contracted, i.e. the gauge index structure of all terms in this line is $\bar{W}^{a} W_{a}$. The third line in (2.14) contains the kinetic term for the real, $\mathrm{SU}\left(N_{B}-1\right)$ neutral field $Z_{\mu}$. The last line in (2.14) contains the only interaction terms present in the Lagrangian. The first interaction term - which will play a crucial role in what follows - has the gauge contraction structure $\bar{W}^{a} A_{a}{ }^{b} W_{b}$. This term completes the derivative in the kinetic part of the $W$ action into an $\mathrm{SU}\left(N_{B}-1\right)$ covariant derivative. It follows, in particular, that the current carried by the $W$ bosons that couples to the $\mathrm{SU}\left(N_{B}-1\right)$ gauge field $A$ is given by

$$
\left(J_{A}^{\mu}\right)_{a}^{b}=\frac{1}{2 \pi} \epsilon^{\mu \nu \rho}\left(W_{\nu}\right)_{a}\left(\bar{W}_{\rho}\right)^{b} .
$$

Finally the second interaction terms in the last line of (2.14) has the gauge structure $Z \bar{W}^{a} W_{a}$. In strictly formal analogy with the discussion above we may define the ' $Z$ boson current'

Note that

$$
J_{Z}^{\mu}=-\frac{1}{2 \pi} \epsilon^{\mu \nu \rho}\left(W_{\nu}\right)_{a}\left(\bar{W}_{\rho}\right)^{a}
$$

$$
J_{Z}^{\mu}=-\operatorname{Tr} J_{A}^{\mu} .
$$

Using these definitions the Lagrangian (2.14) can be rewritten as

$$
\begin{aligned}
S_{\mathrm{E}}= & \frac{i}{4 \pi} \int d^{3} x \operatorname{Tr}\left(\kappa_{B} \epsilon^{\tilde{\mu}-\tilde{\nu}} A_{\tilde{\mu}} \partial_{-} A_{\tilde{\nu}}\right)+\int d^{3} x \bar{W}_{\mu}\left(\frac{i}{2 \pi} \epsilon^{\mu \nu \rho} \partial_{\nu}+\operatorname{sgn}\left(\kappa_{B}\right) v^{2} g^{\mu \rho}\right) W_{\rho} \\
& +\int d^{3} x Z_{\mu}\left(\frac{i \kappa_{B}}{4 \pi} \epsilon^{\mu \nu \rho} \partial_{\nu}+g^{\mu \rho}\left|\kappa_{B}\right| v^{2}\right) Z_{\rho}+\int d^{3} x\left(\operatorname{Tr}\left(A_{\tilde{\mu}} J_{A}^{\tilde{\mu}}\right)+J_{Z}^{\mu} Z_{\mu}\right) .
\end{aligned}
$$

We will find it convenient to work in Fourier space. Our conventions for moving between real and Fourier space are given by

$$
\psi(x)=\int \frac{\mathcal{D}^{3} q}{(2 \pi)^{3}} e^{i x \cdot q} \psi(q),
$$

where $\psi(x)$ is any field and the measure $\mathcal{D}^{3} q$ is defined as follows. In $\mathbb{R}^{3}$ (i.e. when we are interested in zero temperature physics) the integration measure over momenta is usually written as $d^{3} p$. While this measure is perfectly correct as written at zero temperature, at finite temperature we are working on $\mathbb{R}^{2} \times S^{1}$. In this space the measure along $\mathbb{R}^{2}$ is the usual $d p_{1} d p_{2}$, but the measure for the momentum $p_{3}$ along $S^{1}$ which we write as $\mathcal{D} p_{3}$ is different and is given by

$$
\int \mathcal{D} p_{3} f\left(p_{3}\right)=\int_{-\pi}^{\pi} \rho_{B}(\alpha) d \alpha \frac{2 \pi}{\beta} \sum_{n=-\infty}^{\infty} f\left(\frac{2 \pi n+\alpha}{\beta}\right),
$$

\footnotetext{
${ }^{16}$ In this paper we have worked, for definiteness, with the case in which the original gauge group of the bosonic theory is $\mathrm{SU}\left(N_{B}\right)$. Had we instead started with a $\mathrm{U}\left(N_{B}\right)$ bosonic theory, the Lagrangian (2.14) would have continued to apply with the change that $A_{\mu}$ would be a $\mathrm{U}\left(N_{B}-1\right)$ gauge field. All the leading large $N_{B}$ computations and results presented in the rest of this this paper would go through unmodified in this case (because $\mathrm{U}\left(N_{B}-1\right)$ and $\mathrm{SU}\left(N_{B}-1\right)$ results differ only at subleading order in $\left.\frac{1}{N_{B}}\right)$.
} 
where $\rho_{B}(\alpha)$ is the holonomy eigenvalue distribution defined above and $f$ is any function of $p_{3}$. The action (2.18) may then be rewritten as

$$
\begin{aligned}
S_{\mathrm{E}}= & \int \frac{\mathcal{D}^{3} p}{(2 \pi)^{3}} \operatorname{Tr}\left(\frac{A_{\tilde{\mu}}(-p) K^{\tilde{\mu} \tilde{\rho}}(p) A_{\tilde{\rho}}(p)}{2}+J_{A}^{\tilde{\mu}}(-p) A_{\tilde{\mu}}(p)\right) \\
& +\int \frac{\mathcal{D}^{3} p}{(2 \pi)^{3}}\left(\frac{Z_{\mu}(-p) K_{Z}^{\mu \rho}(p) Z_{\rho}(p)}{2}+J_{Z}^{\mu}(-p) Z_{\mu}(p)\right) \\
& +\int \frac{\mathcal{D}^{3} p}{(2 \pi)^{3}} \bar{W}_{\mu}(-p) K_{W}^{\mu \rho}(p) W_{\rho}(p) .
\end{aligned}
$$

where

$$
\begin{aligned}
K^{\tilde{\mu} \tilde{\rho}}(p) & =\frac{-\kappa_{B}}{2 \pi} \epsilon^{\tilde{\mu}-\tilde{\rho}} p_{-}, \\
K_{Z}^{\mu \rho}(p) & =\frac{-\kappa_{B}}{4 \pi} \epsilon^{\mu \nu \rho} p_{\nu}+\left|\kappa_{B}\right| v^{2} g^{\mu \rho}, \\
K_{W}^{\mu \rho}(p) & =-\frac{1}{2 \pi} \epsilon^{\mu \nu \rho} p_{\nu}+\operatorname{sgn}\left(\kappa_{B}\right) v^{2} g^{\mu \rho}, \\
J_{A}^{\tilde{\mu}}(p) & =\frac{1}{2 \pi} \epsilon^{\tilde{\mu} \nu \rho} \int \frac{d^{3} q}{(2 \pi)^{3}} W_{\nu}(p-q) \bar{W}_{\rho}(q), \\
J_{Z}^{\mu}(p) & =-\operatorname{Tr} J_{A}^{\mu}(p) .
\end{aligned}
$$

A path integral based on the action (2.21) can be simplified by integrating out the fields $A_{\mu}$ and $Z_{\mu}$. As each of these fields enter the action (2.21) quadratically, this integrating out procedure can be performed exactly. For each of $A$ and $Z$ we have to complete squares and evaluate a quadratic Gaussian integral. Let us first ignore the determinants and simply classically eliminate the variables $A_{\mu}$ and $Z_{\mu}$ by completing squares. This procedure gives us an effective action for the $W_{\mu}$ and $\bar{W}_{\mu}$ fields given by

$$
\begin{aligned}
S_{\mathrm{E}}[W]= & \int \frac{\mathcal{D}^{3} p}{(2 \pi)^{3}} \bar{W}_{a, \mu}(-p) K_{W}^{\mu \rho}(p) W_{\rho}^{a}(p) \\
& -\frac{1}{2} \int \frac{\mathcal{D}^{3} p}{(2 \pi)^{3}} \frac{\mathcal{D}^{3} q}{(2 \pi)^{3}} \frac{\mathcal{D}^{3} q^{\prime}}{(2 \pi)^{3}}\left[\bar{W}_{\alpha} W_{\beta}\right](q,-p) \Lambda^{\alpha \beta \alpha^{\prime} \beta^{\prime}}\left(q-q^{\prime}, p\right)\left[\bar{W}_{\alpha^{\prime}} W_{\beta^{\prime}}\right]\left(q^{\prime}, p\right),
\end{aligned}
$$

with

$$
\begin{aligned}
K_{\tilde{\mu} \tilde{\nu}}^{-1}(p) & =\frac{2 \pi}{\kappa_{B} p_{-}} \epsilon_{\tilde{\mu}-\tilde{\nu}}, K_{\tilde{\mu} \tilde{\nu}}^{-1}(p) K^{\tilde{\nu} \tilde{\rho}}(p)=\delta_{\tilde{\mu}}^{\tilde{\mu}} \\
K_{Z, \mu \nu}^{-1}(p) & =\frac{-2 \pi m_{Z}}{\left|\kappa_{B}\right|\left(p^{2}+m_{Z}^{2}\right)}\left(\delta_{\mu \nu}-\operatorname{sgn}\left(\kappa_{B}\right) \epsilon_{\mu \nu \rho} \frac{p^{\rho}}{m_{Z}}+\frac{p_{\mu} p_{\nu}}{m_{Z}^{2}}\right), \\
K_{Z, \mu \nu}^{-1}(p) K_{Z}^{\nu \rho}(p) & =\delta_{\mu}^{\rho} \\
\Lambda^{\alpha \beta \alpha^{\prime} \beta^{\prime}}\left(q-q^{\prime}, p\right) & =\Lambda_{A}^{\alpha \beta \alpha^{\prime} \beta^{\prime}}\left(q-q^{\prime}\right)+\Lambda_{Z}^{\alpha \beta \alpha^{\prime} \beta^{\prime}}(p), \\
\Lambda_{A}^{\alpha \beta \alpha^{\prime} \beta^{\prime}}\left(q-q^{\prime}\right) & =\frac{1}{(2 \pi)^{2}} \epsilon^{\beta \alpha^{\prime} \tilde{\mu}} K_{\tilde{\mu} \tilde{\mu}^{\prime}}^{-1}\left(q-q^{\prime}\right) \epsilon^{\tilde{\mu}^{\prime} \beta^{\prime} \alpha} \\
\Lambda_{Z}^{\alpha \beta \alpha^{\prime} \beta^{\prime}}(p) & =\frac{1}{(2 \pi)^{2}} \epsilon^{\alpha \beta \mu} K_{Z, \mu \mu^{\prime}}^{-1}(p) \epsilon^{\mu^{\prime} \alpha^{\prime} \beta^{\prime}}
\end{aligned}
$$


We have used the notation $[B A]$ to denote the singlet combination $B_{a} A^{a}$ where $B$ and $A$ are, respectively, fields that transform in the antifundamental and fundamental of $\mathrm{SU}\left(N_{B}-1\right)$. Moreover the expression $\left[\bar{W}_{\alpha} W_{\beta}\right](q, p)$ in $(2.23)$ is shorthand for

$$
\left[\bar{W}_{\alpha} W_{\beta}\right](q, p) \equiv\left[\bar{W}_{\alpha}\left(q+\frac{p}{2}\right) W_{\beta}\left(-q+\frac{p}{2}\right)\right],
$$

(where $p$ is the centre-of-mass momentum of the bilinear field and $q$ its relative momentum). We note that expressions can be further simplified to give ${ }^{17}$

$$
\begin{aligned}
\Lambda^{\mu \nu \mu^{\prime} \nu^{\prime}}\left(q-q^{\prime}, 0\right)= & \frac{1}{2 \pi \kappa_{B}\left(q-q^{\prime}\right)_{-}}\left(\epsilon^{\nu \mu^{\prime} \nu^{\prime}} \delta_{-}^{\mu}-\epsilon^{\nu \mu^{\prime} \mu} \delta_{-}^{\nu^{\prime}}\right) \\
& -\frac{1}{2 \pi\left|\kappa_{B}\right| m_{Z}}\left(\delta^{\mu \mu^{\prime}} \delta^{\nu \nu^{\prime}}-\delta^{\mu \nu^{\prime}} \delta^{\nu \mu^{\prime}}\right) .
\end{aligned}
$$

For use in the next section we note the some easily verified symmetry properties of the quartic couplings $\Lambda_{A}$ and $\Lambda_{Z}$ above:

$$
\begin{aligned}
& \Lambda_{A}^{\mu \nu \mu^{\prime} \nu^{\prime}}(p)=-\Lambda_{A}^{\mu \nu \mu^{\prime} \nu^{\prime}}(-p)=-\Lambda_{A}^{\mu \mu^{\prime} \nu \nu^{\prime}}(p)=-\Lambda^{\nu^{\prime} \nu \mu^{\prime} \mu}(p), \\
& \Lambda_{Z}^{\mu \nu \mu^{\prime} \nu^{\prime}}(0)=-\Lambda_{Z}^{\nu \mu \mu^{\prime} \nu^{\prime}}(0)=-\Lambda_{Z}^{\mu \nu \nu^{\prime} \mu^{\prime}}(0) .
\end{aligned}
$$

The final path integral we need to perform is given by

$$
\mathcal{Z}=\int[d W] e^{-S_{\mathrm{E}}[W]} \operatorname{det}_{A} \operatorname{det}_{Z}
$$

where $S_{\mathrm{E}}[W]$ is the Euclidean action listed in (2.23), $\operatorname{det}_{A}$ is the determinant that results from integrating out the $A_{\mu}$ fields and $\operatorname{det}_{Z}$ is the determinant resulting from integrating out the $Z_{\mu}$ fields. We now turn to a study of these two determinants.

It is easily verified that

$$
\operatorname{det}_{A}=e^{-\int \frac{\mathcal{D}^{3} p}{(2 \pi)^{3}} \log \frac{i \kappa_{B} p_{-}}{4 \pi}}
$$

This determinant is formally cancelled by the Faddeev Popov determinant associated with the gauge fixing to $A_{-}=0$ and so may be discarded. ${ }^{18}$ On the other hand we find

$$
\operatorname{det}_{Z}=e^{-\frac{1}{2} \int \frac{\mathcal{D}^{3} p}{(2 \pi)^{3}} \log \operatorname{det} K_{Z}^{\nu \rho}(p)}=e^{-\int \frac{\mathcal{V}_{2} d^{2} p_{S}}{(2 \pi)^{2}} \log \left(1-e^{\beta \sqrt{p_{S}^{2}+4 \pi v^{2}}}\right)},
$$

where $\mathcal{V}_{2}$ is the volume of two dimensional space. Eq. (2.29) is a nontrivial function of temperature, but contributes to the logarithm of the partition function only at order unity. The contribution we will obtain below from integrating over the $N_{B} \mathrm{~W}$ bosons will clearly be of order $N_{B}$. Consequently the contribution of (2.29) to the free energy is subleading in an expansion in $\frac{1}{N_{B}}$ and we ignore it in what follows.

\footnotetext{
${ }^{17}$ Here we have used $\epsilon^{\beta \alpha^{\prime}+} \epsilon^{3 \beta^{\prime} \alpha}=\epsilon^{\beta \alpha^{\prime}+}\left(\epsilon^{3+-} \delta_{-}^{\alpha} \delta_{+}^{\beta^{\prime}}+\epsilon^{3-+} \delta_{+}^{\alpha} \delta_{-}^{\beta^{\prime}}\right)$.

${ }^{18} \mathrm{At}$ any event this determinant and its Faddeev-Popov counterpart are both independent of temperature, the $W$ fields and the holonomy fields and so can be absorbed into the normalization of the path integral (equivalently into a shift of the ground state energy) and so can be ignored.
} 
In summary, at leading order in the large $N_{B}$ limit we can simply ignore both determinants $\operatorname{det}_{A}$ and $\operatorname{det}_{Z}$ and work with the simplified path integral

$$
\mathcal{Z}=\int[d W] e^{-S_{\mathrm{E}}[W]}
$$

with $S_{\mathrm{E}}[W]$ given in $(2.23)$. We now proceed to evaluate this path integral in the large $N_{B}$ limit.

\subsection{Dynamics in terms of singlet fields}

In order to exploit the simplifications of the large $N_{B}$ limit we imitate the analysis of [16] and employ a variant of the Hubbard-Stratonovich trick. Specifically we introduce two bilocal but $\mathrm{SU}\left(N_{B}-1\right)$ singlet auxiliary fields $\Sigma^{\mu \nu}(q, p)$ and $\alpha_{\mu \nu}(q, p)$ and introduce these into the path integral using the identities

$$
\begin{aligned}
1 & =\int[d \alpha] \delta\left[\kappa_{B} \alpha_{\mu \nu}(q, p)+\left[\bar{W}_{\mu} W_{\nu}\right](q, p)\right] \\
& =\int[d \alpha][d \Sigma] \exp \left(\int \frac{\mathcal{D}^{3} p}{(2 \pi)^{3}} \frac{\mathcal{D}^{3} q}{(2 \pi)^{3}} i \Sigma^{\nu \mu}(-q,-p)\left(\kappa_{B} \alpha_{\mu \nu}(q, p)+\left[\bar{W}_{\mu} W_{\nu}\right](q, p)\right)\right)
\end{aligned}
$$

Recall the definition of $\left[\bar{W}_{\mu} W_{\nu}\right](q, p)$ from (2.24). Similarly, $p$ is to be thought of as the centre-of-mass momentum and $q$ the relative momentum of the bilocal fields $\alpha_{\mu \nu}(q, p)$ and $\Sigma^{\mu \nu}(q, p)$. Inserting the identity (2.31) into the path integral, the action (2.7) can be written as

$$
\begin{aligned}
\frac{S_{E}[\alpha, \Sigma, W]}{N_{B}}= & -\frac{i}{\lambda_{B}} \int \frac{\mathcal{D}^{3} p}{(2 \pi)^{3}} \frac{\mathcal{D}^{3} q}{(2 \pi)^{3}} \Sigma^{\nu \mu}(q, p) \alpha_{\mu \nu}(-q,-p) \\
& +\frac{1}{N_{B}} \int \frac{\mathcal{D}^{3} q}{(2 \pi)^{3}} \frac{\mathcal{D}^{3} p}{(2 \pi)^{3}} \bar{W}_{\mu}\left(-q-\frac{p}{2}\right) Q^{\mu \nu}(q, p) W_{\nu}\left(q-\frac{p}{2}\right) \\
& -\frac{1}{2 \lambda_{B}} \int \frac{\mathcal{D}^{3} p}{(2 \pi)^{3}} \frac{\mathcal{D}^{3} q}{(2 \pi)^{3}} \frac{\mathcal{D}^{3} q^{\prime}}{(2 \pi)^{3}} \alpha_{\mu \nu}(q,-p) \kappa_{B} \Lambda^{\mu \nu \mu^{\prime} \nu^{\prime}}\left(q-q^{\prime}, p\right) \alpha_{\mu^{\prime} \nu^{\prime}}\left(q^{\prime}, p\right) .
\end{aligned}
$$

where

$$
\begin{aligned}
Q^{\mu \nu}(q, p) & =(2 \pi)^{3} \delta(p) K_{W}^{\mu \nu}(q)-i \Sigma^{\nu \mu}(q, p) \\
\text { or } \quad Q(q, p) & =(2 \pi)^{3} \delta(p) K_{W}(q)-i \Sigma^{T}(q, p) \quad \text { schematically } .
\end{aligned}
$$

and the quartic coupling $\Lambda$ is defined in (2.23). It is useful to define

$$
V[\alpha]=-\frac{1}{2 \lambda_{B}} \int \frac{\mathcal{D}^{3} p}{(2 \pi)^{3}} \frac{\mathcal{D}^{3} q}{(2 \pi)^{3}} \frac{\mathcal{D}^{3} q^{\prime}}{(2 \pi)^{3}} \alpha_{\mu \nu}(q,-p) \kappa_{B} \Lambda^{\mu \nu \mu^{\prime} \nu^{\prime}}\left(q-q^{\prime}, p\right) \alpha_{\mu^{\prime} \nu^{\prime}}\left(q^{\prime}, p\right) .
$$

in terms of which the effective action takes the form

$$
\begin{aligned}
\frac{S_{E}[\alpha, \Sigma, W]}{N_{B}}= & \frac{1}{N_{B}} \int \frac{\mathcal{D}^{3} q}{(2 \pi)^{3}} \frac{\mathcal{D}^{3} p}{(2 \pi)^{3}} \bar{W}_{\mu}\left(-q-\frac{p}{2}\right) Q^{\mu \nu}(q, p) W_{\nu}\left(q-\frac{p}{2}\right) \\
& +V[\alpha]-\frac{i}{\lambda_{B}} \int \frac{\mathcal{D}^{3} p}{(2 \pi)^{3}} \frac{\mathcal{D}^{3} q}{(2 \pi)^{3}} \Sigma^{\nu \mu}(q, p) \alpha_{\mu \nu}(-q,-p) .
\end{aligned}
$$


As the effective action (2.35) is a quadratic function of the $W$-bosons, they can be integrated out. The result of such an integration is an effective action for the bilocal fields that takes the schematic form

$$
S_{\mathrm{eff}}[\alpha, \Sigma]=N_{B}\left(-\frac{i}{\lambda_{B}} \Sigma \cdot \alpha+\log \operatorname{det} Q+V[\alpha]\right) .
$$

As the action (2.36) is of order $N_{B}$ the subsequent integral over the $\Sigma$ and $\alpha$ fields can be performed - at leading order in $1 / N_{B}$ - in the saddle point approximation. We will assume that the saddle point solution for $\Sigma$ and $\alpha$ preserves translational invariance, i.e. that the saddle point solution takes the form

$$
\begin{aligned}
& \Sigma^{\mu \nu}(q, p)=(2 \pi)^{3} \delta(p) \Sigma^{\mu \nu}(q), \\
& \alpha_{\mu \nu}(q, p)=(2 \pi)^{3} \delta(p) \alpha_{\mu \nu}(q) .
\end{aligned}
$$

Under this assumption the expression for $Q^{\mu \nu}(q, p)$ in (2.33) simplifies to

$$
Q(q, p)=(2 \pi)^{3} \delta(p) Q(q), \quad \text { with } \quad Q(q)=K_{W}(q)-i \Sigma^{T}(q) .
$$

From this point on every occurrence of the symbols $\Sigma^{\mu \nu}, \alpha_{\mu \nu}$ and $Q^{\mu \nu}$ in this paper will refer to the 'single momentum' fields on the r.h.s. of (2.37) and (2.38) rather than the bi-momentum field on the l.h.s. of (2.37) and (2.38).

The integral over the $\mathrm{W}$ bosons in (2.32) is now easily performed and gives rise to the following effective action for the $\alpha_{\mu \nu}$ and $\Sigma^{\mu \nu}$ fields:

$$
\frac{S_{\mathrm{eff}}[\alpha, \Sigma]}{N_{B} \mathcal{V}_{3}}=V_{0}[\alpha]-\frac{i}{\lambda_{B}} \int \frac{\mathcal{D}^{3} q}{(2 \pi)^{3}} \Sigma^{\nu \mu}(q) \alpha_{\mu \nu}(-q)+\int \frac{\mathcal{D}^{3} q}{(2 \pi)^{3}} \log \operatorname{det}\left(K_{W}(q)-i \Sigma^{T}(q)\right) .
$$

where $\mathcal{V}_{3}=\mathcal{V}_{2} \beta$ is the volume of spacetime. The quantity $V_{0}[\alpha]$ is obtained by setting the centre-of-mass momentum $p$ to zero in the integrand of $V[\alpha]$ in (2.34) and dividing by $\mathcal{V}_{3}$ :

$$
V_{0}[\alpha]=-\frac{1}{2 \lambda_{B}} \int \frac{\mathcal{D}^{3} q}{(2 \pi)^{3}} \frac{\mathcal{D}^{3} q^{\prime}}{(2 \pi)^{3}} \alpha_{\mu \nu}(q) \kappa_{B} \Lambda^{\mu \nu \mu^{\prime} \nu^{\prime}}\left(q-q^{\prime}, 0\right) \alpha_{\mu^{\prime} \nu^{\prime}}\left(q^{\prime}\right)
$$

\subsection{A symmetry of the gap equations}

Varying the action (2.39) w.r.t. $\Sigma^{\mu \nu}(-q)$ yields the equation

$$
\alpha_{\nu \mu}(q)=\lambda_{B} \frac{\delta}{i \delta \Sigma^{\mu \nu}(-q)} \log \operatorname{det}\left(K_{W}(q)-i \Sigma^{T}(q)\right) .
$$

We might have anticipated from (2.31) that the on-shell value of $\alpha_{\mu \nu}$ would turn out to be the (appropriately normalized and colour stripped) propagator of the $W$ bosons, while $\Sigma^{\mu \nu}$ would turn out to be the self energy in this propagator. This expectation is confirmed by the explicit form of (2.41).

Varying (2.39) w.r.t. the $\alpha_{\mu \nu}(-q)$ yields an expression for the self energy $\Sigma^{\mu \nu}$ in terms of $\alpha_{\mu \nu}$ and so - using (2.41) - in terms of $\Sigma^{\mu \nu}$. Explicitly

$$
\Sigma^{\nu \mu}(q)=\frac{i}{2} \int \frac{\mathcal{D}^{3} q^{\prime}}{(2 \pi)^{3}}\left(\kappa_{B} \Lambda^{\mu \nu \mu^{\prime} \nu^{\prime}}\left(q^{\prime}-q, 0\right)+\kappa_{B} \Lambda^{\mu^{\prime} \nu^{\prime} \mu \nu}\left(q-q^{\prime}, 0\right)\right) \alpha_{\mu^{\prime} \nu^{\prime}}\left(-q^{\prime}\right) .
$$


Before turning to the structure of the r.h.s. of (2.42) in detail, we pause to note an important symmetry property of solutions to this equation. Using the explicit expressions in $(2.22)$ we can immediately verify that

$$
K_{W}^{\mu \nu}(q)=K_{W}^{\nu \mu}(-q), \quad \text { i.e. } \quad K_{W}(q)=K_{W}^{T}(-q) .
$$

It follows that, at tree level,

$$
\alpha(q)_{\mu \nu}=\alpha_{\nu \mu}(-q), \quad \text { i.e. } \quad \alpha(q)=\alpha^{T}(-q)
$$

We will now demonstrate that relations analogous to (2.43) and (2.44) apply not just at tree level but at every order in perturbation theory.

Let us first work at the lowest nontrivial order in perturbation theory. In order to obtain the 'one loop' contribution to the $W$ boson self energy we plug the tree level propagator $\alpha_{\mu \nu}$ (which is of order $\lambda_{B}$ ) into the r.h.s. of the equation (2.45)

$$
\begin{aligned}
\Sigma^{\nu \mu}(q)= & \frac{i}{4 \pi} \int \frac{\mathcal{D}^{3} q^{\prime}}{(2 \pi)^{3}}\left(\epsilon^{\nu \mu^{\prime} \nu^{\prime}} \delta_{-}^{\mu}-\epsilon^{\nu \mu^{\prime} \mu} \delta_{-}^{\nu^{\prime}}-\epsilon^{\nu^{\prime} \mu \nu} \delta_{-}^{\mu^{\prime}}+\epsilon^{\nu^{\prime} \mu \mu^{\prime}} \delta_{-}^{\nu}\right) \frac{\alpha_{\mu^{\prime} \nu^{\prime}}\left(-q^{\prime}\right)}{\left(q^{\prime}-q\right)_{-}}+ \\
& -\frac{i \operatorname{sgn}\left(\kappa_{B}\right)}{2 \pi m_{Z}} \int \frac{\mathcal{D}^{3} q^{\prime}}{(2 \pi)^{3}}\left(\delta^{\mu \mu^{\prime}} \delta^{\nu \nu^{\prime}}-\delta^{\mu \nu^{\prime}} \delta^{\nu \mu^{\prime}}\right) \alpha_{\mu^{\prime} \nu^{\prime}}\left(-q^{\prime}\right) .
\end{aligned}
$$

The resultant expression is of order $\lambda_{B}$ and is the first order (or one loop) correction to $\Sigma^{\mu \nu}$. Using (2.44), it is easy to verify that the second line of $(2.45)$ vanishes. The first line of (2.45) does not vanish and gives a nonzero one loop contribution to $\Sigma^{\mu \nu}$. Using (2.44) however, it is easy to convince oneself that this first order correction to $\Sigma^{\mu \nu}$ obeys

$$
\Sigma^{\mu \nu}(q)=\Sigma^{\nu \mu}(-q), \quad \text { i.e. } \quad \Sigma(q)=\Sigma^{T}(-q) .
$$

It follows that up to first order in $\lambda_{B}$

$$
Q^{\mu \nu}(q)=Q^{\nu \mu}(-q), \quad \text { i.e. } \quad Q(q)=Q^{T}(-q) \text {. }
$$

which implies that $\alpha_{\mu \nu}$ obeys (2.44) up to first subleading order in $\lambda_{B}$.

This argument can now be iterated. In order to obtain the 'two loop' contribution to $\Sigma^{\mu \nu}$ one plugs the $\mathcal{O}\left(\lambda_{B}^{2}\right)$ part of $\alpha_{\mu \nu}$ into the r.h.s. of (2.45) and evaluates the integrals on the r.h.s. The fact that this correction piece in $\alpha_{\mu \nu}$ also obeys (2.44) implies the two loop correction to $\Sigma^{\mu \nu}$ receives no contribution from the second line of (2.45). The entire contribution to this two loop correction comes from the first line of (2.45), which, in turn, now obeys (2.46). It follows that $Q^{\mu \nu}$ obeys (2.47) to second order. This implies $\alpha_{\mu \nu}$ obeys (2.44) upto first subleading order in $\lambda_{B}$ and so on. Iterating the argument above indefinitely we conclude

- The equations $(2.47),(2.44)$ and (2.46) are obeyed at every order in the $\lambda_{B}$ expansion.

- The contribution of second line in (2.45) vanishes at every order in the $\lambda_{B}$ expansion. 


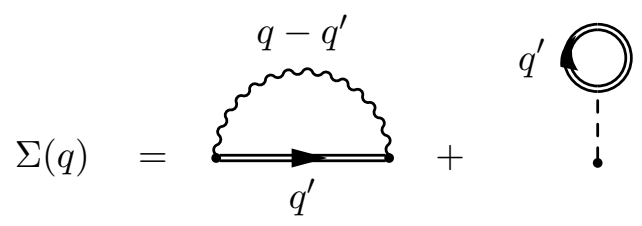

Figure 1. Gap equation.

Note that the second line in (2.45) summarizes the contribution of $Z_{\mu}$ exchange to $\Sigma^{\mu \nu}$. The fact that this line does not contribute to the gap equation at any order in $\lambda_{B}$, and so can just be dropped, tells us that that diagrams involving propagating $Z_{\mu}$ bosons do not contribute to the partition function at leading order in the large $N_{B}$ limit. Note that this conclusion does not follow from large $N_{B}$ counting, but instead follows from the slightly more detailed analysis presented above. ${ }^{19}$

In summary, our saddle-point equations or gap equations take the final form: ${ }^{20}$

$$
\begin{aligned}
& \alpha_{\nu \mu}(q)=\lambda_{B} \frac{\delta}{i \delta \Sigma^{\mu \nu}(-q)} \log \operatorname{det}\left(K_{W}(q)-i \Sigma^{T}(q)\right)=-\lambda_{B}\left(Q^{-1}(q)\right)_{\nu \mu}, \\
& \Sigma^{\nu \mu}(q)=-\frac{i}{4 \pi} \int \frac{\mathcal{D}^{3} q^{\prime}}{(2 \pi)^{3}}\left(\epsilon^{\nu \mu^{\prime} \nu^{\prime}} \delta_{-}^{\mu}-\epsilon^{\nu \mu^{\prime} \mu} \delta_{-}^{\nu^{\prime}}-\epsilon^{\nu^{\prime} \mu \nu} \delta_{-}^{\mu^{\prime}}+\epsilon^{\nu^{\prime} \mu \mu^{\prime}} \delta_{-}^{\nu}\right) \frac{\alpha_{\mu^{\prime} \nu^{\prime}}\left(q^{\prime}\right)}{\left(q^{\prime}+q\right)_{-}} .
\end{aligned}
$$

where $\Sigma^{\mu \nu}, \alpha_{\mu \nu}$ and $Q^{\mu \nu}$ enjoy the symmetry properties (2.46), (2.46) and (2.47) respectively.

Our final gap equation, (2.49), may be diagrammatically summarized as in figure 1. The l.h.s. of the figure is the $W$ boson self energy. On the r.h.s. of the figure the double lines denote the exact $W$ boson propagators, the dashed line is the $Z$ boson propagator while the wiggly line is the $\mathrm{SU}\left(N_{B}-1\right)$ gauge boson propagator. The r.h.s. of (2.49) is entirely captured by the first figure on the r.h.s. of figure 1. This is consistent because the second figure on the r.h.s. of figure 1 (i.e. the contribution to $\Sigma$ of $Z$ boson exchange) vanishes, as we have demonstrated above.

\subsection{Reduction to integral equations of a single variable}

Notice that the r.h.s. of the gap equation (2.49) or (2.55) for $\Sigma(q)$ is independent of $q_{3}$. It follows that each component of $\Sigma^{\mu \nu}$ is independent of $q_{3}$.

The various components of $\Sigma^{\mu \nu}$ can, in general, depend on $q^{+}$and $q^{-}$. To further constrain this dependence we note that our gauge choice $A_{-}=0$ preserves an $\mathrm{SO}(2)$ subgroup of the Euclidean isometry group $\mathrm{SO}(3)$ of our theory. We choose conventions so that $q_{ \pm}$carries unit positive(negative) charge under this subgroup; the general rule is

\footnotetext{
${ }^{19}$ At the diagrammatic level this is the assertion that 'tadpole' contributions to $\Sigma^{\mu \nu}$ — the second graph on the r.h.s. of figure 1 vanishes at all orders.

${ }^{20}$ We have changed variables from $-q^{\prime}$ to $q^{\prime}$ in going from $(2.45)$ to $(2.49)$. To get the second equality in (2.48) we have used that for any non-singular matrix $M$ we have $\log \operatorname{det} M=\operatorname{Tr} \log M \Longrightarrow$ $\delta(\log \operatorname{det} M)=\operatorname{Tr}\left(M^{-1} \delta M\right)$.
} 
that every lower + and upper - sign carries positive unit charge, while every lower - and upper + sign carries negative unit charge. It is easy to verify that, with these conventions, $\mathrm{SO}(2)$ rotations are a symmetry of the gap equations. It follows that any given component of $\Sigma^{\mu \nu}$ must be given by a number of explicit powers of $q_{ \pm}$(determined by the charge of that component) times an unknown function of

$$
w=q_{s}^{2}=2 q_{+} q_{-} .
$$

More specifically, we choose to parametrize non-zero components of $\Sigma^{\mu \nu}$ as

$$
\begin{aligned}
& \Sigma^{--}(q)=\frac{1}{2 \pi q_{-}^{2}} F_{1}(w) \\
& \Sigma^{+-}(q)=+\Sigma^{-+}(q)=\frac{1}{2 \pi} F_{2}(w), \\
& \Sigma^{3-}(q)=-\Sigma^{-3}(q)=\frac{1}{2 \pi q_{-}} F_{3}(w), \\
& \Sigma^{3+}(q)=-\Sigma^{+3}(q)=\frac{q_{-}}{2 \pi} F_{4}(w) .
\end{aligned}
$$

We will now recast the gap equations as equations for four unknown single variable functions $F_{1}, \ldots, F_{4}$.

To start with it is useful to express the matrix $Q^{\mu \nu}$ explicitly in terms of the the functions $F_{1}, \ldots, F_{4}$. We define the quantity $m$ as

$$
m=-\frac{\lambda_{B} m_{B}^{\mathrm{cri}}}{2}=\operatorname{sgn}\left(\kappa_{B}\right) 2 \pi v^{2}
$$

where we have used (2.3) to get the second equality above (see also (A.13) of appendix A). Note that $|m|=\left|m_{W}\right|$ (see (2.11)) and also the fact that $m$ changes sign as $\kappa_{B}$ changes sign. With this notation we find the quadratic kernel $Q^{\mu \nu}(q)$ as defined in (2.38) (the matrix is presented in the order,,+- 3 )

$$
Q^{\mu \nu}(q)=\frac{1}{2 \pi}\left[\begin{array}{ccc}
0 & -i\left(F_{2}+i m+q_{3}\right) & i q_{-}\left(1-F_{4}\right) \\
-i\left(F_{2}+i m-q_{3}\right) & -\frac{i}{q_{-}^{2}} F_{1}(w) & -\frac{i}{q_{-}}\left(F_{3}+\frac{w}{2}\right) \\
-i q_{-}\left(1-F_{4}\right) & \frac{i}{q_{-}}\left(F_{3}+\frac{w}{2}\right) & m
\end{array}\right]
$$

The zeros of determinant of the matrix $Q^{\mu \nu}$ are the pole mass of the propagator. The determinant is given by

$$
\begin{aligned}
\operatorname{det} Q & =-\frac{m}{8 \pi^{3}}\left(q^{2}+M^{2}(w)\right) \\
q^{2} & =w+q_{3}^{2} \\
M^{2}(w) & =-\left(F_{2}+i m\right)^{2}-\frac{i}{m} F_{1}\left(1-F_{4}\right)^{2}-\frac{i}{m}\left(F_{2}+i m\right)\left(w+2 F_{3}\right)\left(1-F_{4}\right)-w .
\end{aligned}
$$

With these formulae at hand now we proceed to give explicit form of the gap equations for $\Sigma, \alpha$. 
The gap equation (2.49) is given by

$$
\begin{aligned}
\Sigma^{33}(q) & =0 \\
\Sigma^{++}(q) & =0 \\
\Sigma^{--}(q) & =-\frac{1}{\pi} \int \frac{\mathcal{D}^{3} q^{\prime}}{(2 \pi)^{3}} \frac{1}{\left(q+q^{\prime}\right)_{-}} \alpha_{3+}\left(q^{\prime}\right) \\
\Sigma^{+-}(q) & =-\frac{1}{2 \pi} \int \frac{\mathcal{D}^{3} q^{\prime}}{(2 \pi)^{3}} \frac{1}{\left(q+q^{\prime}\right)_{-}} \alpha_{-3}\left(q^{\prime}\right), \\
\Sigma^{3-}(q) & =\frac{1}{2 \pi} \int \frac{\mathcal{D}^{3} q^{\prime}}{(2 \pi)^{3}} \frac{1}{\left(q+q^{\prime}\right)_{-}} \alpha_{-+}\left(q^{\prime}\right), \\
\Sigma^{3+}(q) & =-\frac{1}{2 \pi} \int \frac{\mathcal{D}^{3} q^{\prime}}{(2 \pi)^{3}} \frac{1}{\left(q+q^{\prime}\right)_{-}} \alpha_{--}\left(q^{\prime}\right) .
\end{aligned}
$$

It follows from (2.48) that the components of $\alpha(q)=-\lambda_{B} Q(q)^{-1}$ are

$$
\begin{aligned}
& \alpha_{++}(q)=\frac{\lambda_{B}}{(2 \pi)^{2} \operatorname{det} Q} \frac{1}{q_{-}^{2}}\left(i m F_{1}+\left(F_{3}+\frac{w}{2}\right)^{2}\right), \\
& \alpha_{-+}(q)=\frac{\lambda_{B}}{(2 \pi)^{2} \operatorname{det} Q}\left(\left(1-F_{4}\right)\left(F_{3}+\frac{w}{2}\right)-i m\left(F_{2}+i m-q_{3}\right)\right), \\
& \alpha_{--}(q)=\frac{\lambda_{B}}{(2 \pi)^{2} \operatorname{det} Q} q_{-}^{2}\left(1-F_{4}\right)^{2}, \\
& \alpha_{-3}(q)=-\frac{\lambda_{B}}{(2 \pi)^{2} \operatorname{det} Q} q_{-}\left(1-F_{4}\right)\left(F_{2}+i m-q_{3}\right), \\
& \alpha_{3+}(q)=-\frac{\lambda_{B}}{(2 \pi)^{2} \operatorname{det} Q} \frac{1}{q_{-}}\left(F_{1}\left(1-F_{4}\right)+\left(F_{2}+i m-q_{3}\right)\left(F_{3}+\frac{w}{2}\right)\right), \\
& \alpha_{33}(q)=-\frac{\lambda_{B}}{(2 \pi)^{2} \operatorname{det} Q}\left(\left(F_{2}+i m\right)^{2}-q_{3}^{2}\right) .
\end{aligned}
$$

Inserting (2.56) into (2.49) we find the explicit coupled integral equations:

$$
\begin{aligned}
\frac{1}{q_{-}^{2}} F_{1}(w) & =\frac{\lambda_{B}}{(2 \pi)^{2}} \int \frac{\mathcal{D}^{3} q^{\prime}}{(2 \pi)^{3}} \frac{2 F_{1}\left(1-F_{4}\right)+\left(F_{2}+i m\right)\left(2 F_{3}+w^{\prime}\right)}{\operatorname{det} Q\left(q^{\prime}\right)\left(q+q^{\prime}\right)_{-} q_{-}^{\prime}} \\
F_{2}(w) & =\frac{\lambda_{B}}{(2 \pi)^{2}} \int \frac{\mathcal{D}^{3} q^{\prime}}{(2 \pi)^{3}} \frac{\left(1-F_{4}\right)\left(F_{2}+i m-q_{3}^{\prime}\right) q_{-}^{\prime}}{\operatorname{det} Q\left(q^{\prime}\right)\left(q+q^{\prime}\right)_{-}} \\
\frac{1}{q_{-}} F_{3}(w) & =\frac{\lambda_{B}}{(2 \pi)^{2}} \int \frac{\mathcal{D}^{3} q^{\prime}}{(2 \pi)^{3}} \frac{\left(F_{3}+\frac{w^{\prime}}{2}\right)\left(1-F_{4}\right)-i m\left(F_{2}+i m-q_{3}^{\prime}\right)}{\operatorname{det} Q\left(q^{\prime}\right)\left(q+q^{\prime}\right)_{-}} \\
q_{-} F_{4}(w) & =-\frac{\lambda_{B}}{(2 \pi)^{2}} \int \frac{\mathcal{D}^{3} q^{\prime}}{(2 \pi)^{3}} \frac{\left(1-F_{4}\right)^{2} q_{-}^{\prime 2}}{\operatorname{det} Q\left(q^{\prime}\right)\left(q+q^{\prime}\right)_{-}} .
\end{aligned}
$$

(All the functions $F_{1}, F_{2}, F_{3}$ and $F_{4}$ on the r.h.s. of (2.57) and have the argument $w^{\prime}$.) 
Substituting the expression for $\operatorname{det} Q$ from (2.54) we obtain

$$
\begin{aligned}
\frac{1}{q_{-}^{2}} F_{1}(w) & =-\frac{2 \pi \lambda_{B}}{m} \int \frac{\mathcal{D}^{3} q^{\prime}}{(2 \pi)^{3}} \frac{2 F_{1}\left(1-F_{4}\right)+\left(F_{2}+i m\right)\left(2 F_{3}+w^{\prime}\right)}{\left(\left(q_{3}^{\prime}\right)^{2}+w^{\prime}+M^{2}\left(w^{\prime}\right)\right)\left(\left(q+q^{\prime}\right)_{-} q_{-}^{\prime}\right)}, \\
F_{2}(w) & =-\frac{2 \pi \lambda_{B}}{m} \int \frac{\mathcal{D}^{3} q^{\prime}}{(2 \pi)^{3}} \frac{\left(1-F_{4}\right)\left(F_{2}+i m-q_{3}^{\prime}\right) q_{-}^{\prime}}{\left(\left(q_{3}^{\prime}\right)^{2}+w^{\prime}+M^{2}\left(w^{\prime}\right)\right)\left(q+q^{\prime}\right)_{-}}, \\
\frac{1}{q_{-}} F_{3}(w) & =-\frac{2 \pi \lambda_{B}}{m} \int \frac{\mathcal{D}^{3} q^{\prime}}{(2 \pi)^{3}} \frac{\left(F_{3}+\frac{w^{\prime}}{2}\right)\left(1-F_{4}\right)-i m\left(F_{2}+i m-q_{3}^{\prime}\right)}{\left(\left(q_{3}^{\prime}\right)^{2}+w^{\prime}+M^{2}\left(w^{\prime}\right)\right)\left(q+q^{\prime}\right)_{-}}, \\
q_{-} F_{4}(w) & =\frac{2 \pi \lambda_{B}}{m} \int \frac{\mathcal{D}^{3} q^{\prime}}{(2 \pi)^{3}} \frac{\left(1-F_{4}\right)^{2} q_{-}^{\prime 2}}{\left(\left(q_{3}^{\prime}\right)^{2}+w^{\prime}+M^{2}\left(w^{\prime}\right)\right)\left(q+q^{\prime}\right)_{-}} .
\end{aligned}
$$

The dependence of the integrands on the r.h.s. of (2.58) on $q_{3}^{\prime}$ is completely explicit since the unknown functions on the r.h.s. are all functions of $w^{\prime}$. As a consequence the integral (sum) over $q_{3}^{\prime}$ is easily evaluated as we now demonstrate. Recall, from equation (2.20), that the 'integral over $q_{3}$ ' is really a discrete sum

$$
\int \frac{\mathcal{D} q_{3}}{2 \pi} f\left(q_{3}\right)=\int d \alpha \rho_{B}(\alpha) \beta^{-1} \sum_{n \in \mathbb{Z}} f\left(\frac{\alpha+2 \pi n}{\beta}\right) .
$$

To proceed, we make the assumption that the holonomy distribution $\rho_{B}(\alpha)$ is an even function of $\alpha$ :

$$
\rho_{B}(\alpha)=\rho_{B}(-\alpha)
$$

It follows that all integrals with an odd number of $q_{3}^{\prime}$ factors vanish in (2.58). In terms of the function $\chi(z)$ defined as

$$
\begin{aligned}
\chi(z) \equiv & -\frac{(2 \pi)^{3}}{m \beta} \int d \alpha \rho_{B}(\alpha) \sum_{n \in \mathbb{Z}} \frac{1}{\left(2 \pi \frac{n}{\beta}+\frac{\alpha}{\beta}\right)^{2}+\left(z+M^{2}(z)\right)}, \\
= & -\frac{2 \pi^{3}}{m} \int d \alpha \rho_{B}(\alpha) \frac{1}{\sqrt{z+M^{2}(z)}} \times \\
& \times\left(\operatorname{coth}\left(\frac{\beta}{2}\left(\sqrt{z+M^{2}(z)}+i \frac{\alpha}{\beta}\right)\right)+\operatorname{coth}\left(\frac{\beta}{2}\left(\sqrt{z+M^{2}(z)}-i \frac{\alpha}{\beta}\right)\right)\right),
\end{aligned}
$$

the integral equations (2.58) become

$$
\begin{aligned}
\frac{1}{q_{-}^{2}} F_{1}(w) & =\frac{\lambda_{B}}{(2 \pi)^{2}} \int \frac{d^{2} q^{\prime}}{(2 \pi)^{2}} \chi\left(w^{\prime}\right) \frac{2 F_{1}\left(1-F_{4}\right)+\left(F_{2}+i m\right)\left(2 F_{3}+w^{\prime}\right)}{\left(q+q^{\prime}\right)_{-} q_{-}^{\prime}}, \\
F_{2}(w) & =\frac{\lambda_{B}}{(2 \pi)^{2}} \int \frac{d^{2} q^{\prime}}{(2 \pi)^{2}} \chi\left(w^{\prime}\right) \frac{\left(1-F_{4}\right)\left(F_{2}+i m\right) q_{-}^{\prime}}{\left(q+q^{\prime}\right)_{-}}, \\
\frac{1}{q_{-}} F_{3}(w) & =\frac{\lambda_{B}}{(2 \pi)^{2}} \int \frac{d^{2} q^{\prime}}{(2 \pi)^{2}} \chi\left(w^{\prime}\right) \frac{\left(F_{3}+\frac{w^{\prime}}{2}\right)\left(1-F_{4}\right)-i m\left(F_{2}+i m\right)}{\left(q+q^{\prime}\right)_{-}}, \\
q_{-} F_{4}(w) & =-\frac{\lambda_{B}}{(2 \pi)^{2}} \int \frac{d^{2} q^{\prime}}{(2 \pi)^{2}} \chi\left(w^{\prime}\right) \frac{\left(1-F_{4}\right)^{2} q_{-}^{\prime 2}}{\left(q+q^{\prime}\right)_{-}} .
\end{aligned}
$$


In the next section we will frequently require the (indefinite) integral of $\chi(z)$ defined in (2.61) with respect to $z$. This integral is easily evaluated; we find

$$
\begin{aligned}
\xi(z)= & -\frac{1}{2(2 \pi)^{3}} \int^{z} d w^{\prime} \chi\left(w^{\prime}\right) \\
= & \frac{1}{2 m \beta} \int d \alpha \rho_{B}(\alpha)\left[\log 2 \sinh \left(\frac{\beta}{2}\left(\sqrt{z+M^{2}(z)}+i \frac{\alpha}{\beta}\right)\right)+\right. \\
& \left.+\log 2 \sinh \left(\frac{\beta}{2}\left(\sqrt{z+M^{2}(z)}-i \frac{\alpha}{\beta}\right)\right)\right]
\end{aligned}
$$

\subsection{Dimensional regularization}

Later in this section we will encounter divergent integrals that will need to be regulated. Following [1] we will perform this regulation by employing dimensionally regulated version of the summation in (2.61) which effectively replaces (2.61) by (see around Sec (2.2) of [1] for details)

$$
\begin{aligned}
\chi(z)= & -\frac{2 \pi^{3}}{m} \int d \alpha \rho_{B}(\alpha) \frac{1}{\left(\sqrt{z+M^{2}(z)}\right)^{1+\epsilon}} \times \\
& \times\left[\operatorname{coth}\left(\frac{\beta}{2}\left(\sqrt{z+M^{2}(z)}+i \frac{\alpha}{\beta}\right)\right)+\operatorname{coth}\left(\frac{\beta}{2}\left(\sqrt{z+M^{2}(z)}-i \frac{\alpha}{\beta}\right)\right)\right],
\end{aligned}
$$

and effectively replaces (2.63) by

$$
\begin{aligned}
\xi(z)= & \frac{1}{2 m \beta} \int d \alpha \rho_{B}(\alpha) \frac{1}{\left(\sqrt{z+M^{2}(z)}\right)^{\epsilon}} \times \\
& \times\left[\log 2 \sinh \left(\frac{\beta}{2}\left(\sqrt{z+M^{2}(z)}+i \frac{\alpha}{\beta}\right)\right)+\log 2 \sinh \left(\frac{\beta}{2}\left(\sqrt{z+M^{2}(z)}-i \frac{\alpha}{\beta}\right)\right)\right],
\end{aligned}
$$

where $\epsilon$ is an infinitesimal that is taken to zero at the end of the computation (there are additional terms in the indefinite integral of (2.64) which are proportional to $\epsilon$. However, since we always take the limit $\epsilon \rightarrow 0$, we drop these extra terms from (2.65)).

\subsection{Performing the angular integrals}

While the r.h.s. of (2.62) is given in terms of two dimensional integrals $d^{2} q^{\prime}$ the unknown functions on the r.h.s. are functions only of $w^{\prime}$. For this reason we can explicitly perform all angular integrals in the r.h.s. of the four equations (2.62). Let $q_{-}^{\prime}=\frac{1}{\sqrt{2}} q_{s}^{\prime} e^{i \eta}$. It is easy to check that

$$
\begin{aligned}
\int_{0}^{2 \pi} \frac{d \eta}{2 \pi} \frac{1}{q_{-}+q_{-}^{\prime}} & =\frac{1}{q_{-}} \Theta\left(q_{s}-q_{s}^{\prime}\right), & \int_{0}^{2 \pi} \frac{d \eta}{2 \pi} \frac{1}{\left(q_{-}+q_{-}^{\prime}\right) q_{-}^{\prime}} & =-\frac{1}{q_{-}^{2}} \Theta\left(q_{s}-q_{s}^{\prime}\right) \\
\int_{0}^{2 \pi} \frac{d \eta}{2 \pi} \frac{q_{-}^{\prime}}{q_{-}+q_{-}^{\prime}} & =\Theta\left(q_{s}^{\prime}-q_{s}\right), & \int_{0}^{2 \pi} \frac{d \eta}{2 \pi} \frac{q_{-}^{\prime 2}}{q_{-}+q_{-}^{\prime}} & =-q_{-} \Theta\left(q_{s}^{\prime}-q_{s}\right) .
\end{aligned}
$$


Using these results to perform all angular integrals on the r.h.s. of (2.62) we find that the integral equations take the form

$$
\begin{aligned}
& F_{1}(w)=-\frac{\lambda_{B}}{(2 \pi)^{2}} \int_{0}^{w} \frac{d w^{\prime}}{4 \pi} \chi\left(w^{\prime}\right)\left(2 F_{1}\left(1-F_{4}\right)+\left(F_{2}+i m\right)\left(2 F_{3}+w^{\prime}\right)\right), \\
& F_{2}(w)=\frac{\lambda_{B}}{(2 \pi)^{2}} \int_{w}^{\infty} \frac{d w^{\prime}}{4 \pi} \chi\left(w^{\prime}\right)\left(1-F_{4}\right)\left(F_{2}+i m\right), \\
& F_{3}(w)=\frac{\lambda_{B}}{(2 \pi)^{2}} \int_{0}^{w} \frac{d w^{\prime}}{4 \pi} \chi\left(w^{\prime}\right)\left(\left(F_{3}+\frac{w^{\prime}}{2}\right)\left(1-F_{4}\right)-i m\left(F_{2}+i m\right)\right), \\
& F_{4}(w)=\frac{\lambda_{B}}{(2 \pi)^{2}} \int_{w}^{\infty} \frac{d w^{\prime}}{4 \pi} \chi\left(w^{\prime}\right)\left(1-F_{4}\right)^{2}
\end{aligned}
$$

\subsection{Differential equations for the unknowns}

We obtain the following differential equations for the functions $F_{1}(w), \ldots, F_{4}(w)$ by differentiating them with respect to their arguments:

$$
\begin{aligned}
& F_{1}^{\prime}(w)=-\frac{\lambda_{B}}{2(2 \pi)^{3}} \chi(w)\left(2 F_{1}\left(1-F_{4}\right)+\left(F_{2}+i m\right)\left(2 F_{3}+w\right)\right), \\
& F_{2}^{\prime}(w)=-\frac{\lambda_{B}}{2(2 \pi)^{3}} \chi(w)\left(1-F_{4}\right)\left(F_{2}+i m\right), \\
& F_{3}^{\prime}(w)=\frac{\lambda_{B}}{2(2 \pi)^{3}} \chi(w)\left(\left(F_{3}+\frac{w}{2}\right)\left(1-F_{4}\right)-i m\left(F_{2}+i m\right)\right), \\
& F_{4}^{\prime}(w)=-\frac{\lambda_{B}}{2(2 \pi)^{3}} \chi(w)\left(1-F_{4}\right)^{2} .
\end{aligned}
$$

Now it follows from the definition of $M^{2}(w)$ in (2.54) that when (2.69) are satisfied

$$
M^{\prime}(w)=-\frac{1}{2 M(w)}\left(F_{4}(w)+\frac{i F_{2}(w)\left(1-F_{4}(w)\right)}{m}\right) .
$$

We will return to this equation in a bit.

\subsection{Solving the gap equations}

\subsubsection{Determining $F_{4}$}

The integral equation for the function $F_{4}$ (see (2.68)) can be solved very simply in multiple different ways. To begin with we solve this equation order by order in perturbation theory. We proceed by expanding $F_{4}$ in a perturbative expansion in $\lambda_{B}$

$$
F_{4}=f_{0}+\lambda_{B} f_{1}+\lambda_{B}^{2} f_{2}+\cdots .
$$

and simply plug this ansatz back in to the integral equation. The equation takes the form

$$
f_{0}+\lambda_{B} f_{1}+\lambda_{B}^{2} f_{2}+\cdots=\frac{\lambda_{B}}{2(2 \pi)^{3}} \int_{w}^{\infty} d w^{\prime} \chi\left(w^{\prime}\right)\left(f_{0}-1+\lambda_{B} f_{1}+\lambda_{B}^{2} f_{2}+\cdots\right)^{2} .
$$

and yields the following infinite sequence of equations

$$
f_{0}=0, \quad f_{1}=\frac{1}{2(2 \pi)^{3}} \int_{w}^{\infty} d w^{\prime} \chi\left(w^{\prime}\right), \quad f_{2}=-\frac{1}{2(2 \pi)^{3}} \int_{w}^{\infty} d w^{\prime} \chi\left(w^{\prime}\right) 2 f_{1}\left(w^{\prime}\right), \ldots
$$


Each of these equations can be solved in a straightforward manner by integration. The only subtlety here is that, at every order, the indefinite integrals in question diverge at large $w^{\prime}$. We use the dimensional regulation scheme outlined in subsection 2.6 to define these divergent integrals. In order to proceed with our analysis we assume that the mass parameter $M(z)$ tends to a constant $M$ at least at large $z$; in the next subsection we will demonstrate that this assumption is self-consistent.

In order to see how this works lets start with the second equation in (2.73). It follows immediately from the definition (2.63) that

$$
f_{1}=\xi(w)-\xi(\infty) .
$$

The problem with $(2.74)$ is that $\xi(\infty)$ is divergent; indeed it is easily verified from the regularized version (2.65) that at large $w$

$$
\xi(w) \rightarrow \frac{1}{2 m}\left(\sqrt{w+M^{2}(w)}\right)^{1-\epsilon} \equiv \xi_{\text {asymp }}(w) .
$$

In order to make sense of (2.74) we proceed as follows. Consider the function $B(w)$

$$
B(w)=\frac{1-\epsilon}{4 m\left(\sqrt{w+M^{2}(w)}\right)^{1+\epsilon}}
$$

$B(z)$ is defined so as to obey the identity

$$
B(z)=\xi_{\text {asymp }}^{\prime}(z)
$$

Now we evaluate the integral for $f_{1}(w)$ in $(2.73)$ as follows:

$$
-2(2 \pi)^{3} f_{1}=\int_{w}^{\infty} d w^{\prime}\left(\chi\left(w^{\prime}\right)-B\left(w^{\prime}\right)\right)+\int_{w}^{\infty} B\left(w^{\prime}\right)
$$

The first integral in (2.76) is now convergent and evaluates to

$$
-\xi(w)+\xi_{\text {asymp }}(w),
$$

(note that the contributions at infinity cancel). The second integral in (2.76) is divergent and is evaluated using dimensional regularization. It evaluates to

$$
-\xi_{\text {asymp }}(w) \text {. }
$$

Adding together the two terms we find the well defined expression

$$
f_{1}(w)=\xi(w) .
$$

Notice that the net effect of our dimensional regulation scheme was to simply drop the surface term at infinity. It is easy to convince oneself that this scheme effectively does the same thing (i.e. drops all surface terms at infinity) in all the integrals that appear in the perturbative evaluation of $F_{4}$. Adopting this prescription we find

$$
\begin{aligned}
F_{4}(w) & =\lambda_{B} \xi(w)-\lambda_{B}^{2} \xi(w)^{2}+\lambda_{B}^{3} \xi(w)^{3}-\cdots \\
& =1-\sum_{n=0}^{\infty}\left(-\lambda_{B}\right)^{n} \xi(w)^{n}=1-\frac{1}{1+\lambda_{B} \xi(w)} .
\end{aligned}
$$


Summing up, we have

$$
1-F_{4}=\frac{1}{1+\lambda_{B} \xi(w)}
$$

As a consistency check, it is easy to verify that our solution (2.79) obeys its differential equation (fourth of (2.69)). Indeed this differential equation is easy to solve in generality; its most general solution is

$$
\frac{1}{1-F_{4}(w)}=\lambda_{B} \xi(w)+c_{4}
$$

where $c_{4}$ is an integration constant. Clearly (2.80) reduces to (2.79) if we choose

$$
c_{4}=1 \text {. }
$$

\subsubsection{A subtlety in $F_{4}$}

In order to complete the process of checking our solution, let us directly check that the solution (2.79) obeys the integral equation (2.68) which we reproduce here for clarity.

$$
F_{4}(w)=\frac{\lambda_{B}}{(2 \pi)^{2}} \int_{w}^{\infty} \frac{d w^{\prime}}{4 \pi} \chi\left(w^{\prime}\right)\left(1-F_{4}\right)^{2}
$$

We will find that this check helps us better understand the procedure we used to obtain the solution (2.79), by contrasting it with an equally reasonable sounding procedure that does not work.

Let $F_{4}$ be any solution of the fourth of (2.69), i.e. a solution of the form (2.80) with any value of $c_{4}$. For every such solution

$$
\left(1-F_{4}\right)^{2}=\frac{1}{\left(\lambda_{B} \xi(w)+c_{4}\right)^{2}}
$$

Inserting this into the r.h.s. of (2.82) and using the fact that

$$
\chi(w) d w=-2(2 \pi)^{3} d \xi
$$

we conclude that the r.h.s. of (2.82) evaluates to

$$
-\frac{1}{\lambda_{B} \xi(w)+c_{4}}=F_{4}-1
$$

(where we have used the fact that $\xi$ diverges as $w \rightarrow \infty$ - note in particular that the integral on the r.h.s. of (2.82) is convergent). On the other hand the l.h.s. of (2.82) is $F_{4}$. As $F_{4} \neq F_{4}-1$ we find that the r.h.s. and l.h.s. of these equations do not agree for any value of $c_{4}$. It follows, in other words, that no solution of the differential equations (2.69) obeys the integral equation (2.82). As we have earlier argued that every solution of the integral equations (e.g. (2.82)) obey the differential equations (2.69), we are forced to conclude that the integral equation (2.82) has no solutions!

The conclusion of the previous paragraph appears to be in direct conflict with the fact that - in the previous subsubsection - we have actually found an explicit solution namely (2.79) — of the equation (2.82). To make this contradiction as sharp as possible 
let us specialize the analysis in the paragraph around (2.83) to the special case $c_{4}=1$. In this case the solution presented in (2.83) is the perturbative solution (2.79). How can it be that the analysis in the paragraph around (2.83) demonstrates that this solution does not obey the integral equation (2.82), while the analysis earlier in this subsection demonstrates that it does?

The answer to this question is simply that the expansion of the quantity $\left(1-F_{4}\right)^{2}$ on the r.h.s. of (2.82) in a power series in $\lambda_{B}$ does not commute with the integral over $w^{\prime}$ in (2.82). More precisely let us contrast two methods of evaluating the integral (2.82) that give different answers.

The first method - the one adopted in this subsubsection - is to performing the sum over $\lambda_{B}$ first (as in (2.78)), then to notice that the resultant integrand defines a convergent integral in (2.82), and to evaluate the integral.

The second method - adopted in subsubsection 2.9.1 - on the other hand, is to first expand the integrand on the r.h.s. of (2.82) in a power series in $\lambda_{B}$, perform the integral order by order for each of the coefficients of $\lambda_{B}^{n}$ and then to sum the final power series of results. Crucially the integrals encountered at every order in the $\lambda_{B}$ expansion are divergent and need to be defined. Defining the integrals by a form of dimensional regularization yields a result for the r.h.s. that agrees with the l.h.s. of (2.82).

The second method is guaranteed to reproduce the results of Feynman diagram based perturbation theory (because it simply is the integral equation's way of generating Feynman diagrams loop by loop). As we require all our results to agree with perturbation theory we will take the view that the second method is the correct one all through this paper, and so (2.79) is the correct solution for $F_{4}$.

We will not encounter similar subtleties in any of the other integral equations in this paper.

\subsubsection{A curious observation}

In this subsection we note a curious fact relating to the subtlety of the last subsection. It turns out that there is a second, apparently ad hoc - but nonetheless interesting procedure that yields the same answer for our $W$ boson propagator as the procedure outlined in the previous subsection and employed in the rest of this paper. This subsection is devoted to a description of this alternate procedure.

The analysis of this subsection will be used no where else in this paper - and may turn out to be a curiosity with no deeper significance. The impatient reader should feel free to skip over to the next subsection.

The ad hoc procedure we will employ in this subsection is to modify our starting action - (2.18) - in the manner that we now describe: we simply drop the term proportional to $W_{+} \partial_{-} W_{3}$ that occurs in the expansion of the first term in the second line of (2.18). If we then rerun the analysis of this paper but starting with this modified action we find, in particular, that

$$
Q^{\mu \nu}(q)=\frac{1}{2 \pi}\left[\begin{array}{ccc}
0 & -i\left(F_{2}+i m+q_{3}\right) & i q_{-}\left(-\tilde{F}_{4}\right) \\
-i\left(F_{2}+i m-q_{3}\right) & -\frac{i}{q_{-}^{2}} F_{1}(w) & -\frac{i}{q_{-}}\left(F_{3}+\frac{w}{2}\right) \\
-i q_{-}\left(-\tilde{F}_{4}\right) & \frac{i}{q_{-}}\left(F_{3}+\frac{w}{2}\right) & m
\end{array}\right] .
$$


where $\tilde{F}_{4}$ parametrizes the self energy contribution to $\Sigma^{3+}$ in the modified problem, in exactly the same way that $F_{4}$ parametrized the same quantity in the original problem. ${ }^{21}$ Proceeding as above, we find that our modified problem leads to the integral equations

$$
\begin{aligned}
& F_{1}(w)=-\frac{\lambda_{B}}{(2 \pi)^{2}} \int_{0}^{w} \frac{d w^{\prime}}{4 \pi} \chi\left(w^{\prime}\right)\left(2 F_{1}\left(-\tilde{F}_{4}\right)+\left(F_{2}+i m\right)\left(2 F_{3}+w^{\prime}\right)\right), \\
& F_{2}(w)=\frac{\lambda_{B}}{(2 \pi)^{2}} \int_{w}^{\infty} \frac{d w^{\prime}}{4 \pi} \chi\left(w^{\prime}\right)\left(-\tilde{F}_{4}\right)\left(F_{2}+i m\right), \\
& F_{3}(w)=\frac{\lambda_{B}}{(2 \pi)^{2}} \int_{0}^{w} \frac{d w^{\prime}}{4 \pi} \chi\left(w^{\prime}\right)\left(\left(F_{3}+\frac{w^{\prime}}{2}\right)\left(-\tilde{F}_{4}\right)-i m\left(F_{2}+i m\right)\right), \\
& \tilde{F}_{4}(w)=\frac{\lambda_{B}}{(2 \pi)^{2}} \int_{w}^{\infty} \frac{d w^{\prime}}{4 \pi} \chi\left(w^{\prime}\right)\left(-\tilde{F}_{4}\right)^{2} .
\end{aligned}
$$

In particular the last of (2.86) is easily solved at finite $\lambda_{B}$ (there is not need to expand in $\lambda_{B}$ before performing the integrals) and we find

$$
\tilde{F}_{4}=F_{4}-1=-\frac{1}{1+\lambda_{B} \xi(w)}
$$

Comparing (2.85), (2.53) and (2.87), it follows that $Q^{\mu \nu}$ of this subsection is now identical - as a function of $F_{2}, F_{3}$ and $F_{1}$ - to $Q^{\mu \nu}$ of the actual problem. The remaining integral equations of modified problem - the first three of (2.86) with (2.87) plugged in - are now identical to the integral equations (2.68) of the original problem with (2.79) plugged in. It follows, in particular, that from this point on, the equations for the two problems are the same.

Let us summarize. There are two procedures that yield the same thermal propagator. The first uses the actual classical action of our system as its starting point but evaluates all integrals by expanding out the integrands term by term in an expansion in $\lambda_{B}$ and then evaluating the integrals that appear at each order using the dimensional regularization scheme. This is the procedure adopted in earlier subsections and in the rest of this paper. In the second procedure we evaluate all integrals first (before performing any expansions in $\lambda_{B}$ that may be of interest). The second procedure gives us the same result as the first, if we modify the starting action with a very particular 'counterterm'.

In other words it appears that the two different regulation schemes (using dimensional regularization before or after expanding in $\lambda_{B}$ ) differ by a very particular counterterm. It is, of course, usual for different regulation schemes to effectively differ by counterterms. The novelty in the current situation is that the needed counterterm occurs at leading (classical) order in the loop expansion rather than at higher orders as is more usual.

\subsubsection{Determining $F_{2}$}

We will now determine the function $F_{2}$. The differential equation for $F_{2}$ is given by

$$
F_{2}^{\prime}(w)=-\frac{\lambda_{B}}{2(2 \pi)^{3}} \chi(w)\left(1-F_{4}\right)\left(F_{2}+i m\right) .
$$

\footnotetext{
${ }^{21}$ In principle we should also replace $F_{2}$ by $\tilde{F}_{2}$, and similarly for $F_{3}$ and $F_{1}$, but it will turn out below that $\tilde{F}_{i}=F_{i}$ for $i=1 \ldots 3$, so we will avoid cluttering the notation.
} 
Plugging in the expression for $1-F_{4}$ from $(2.79)$ and using $(2.63)$, we have

$$
\frac{d\left(F_{2}+i m\right)}{F_{2}+i m}=\frac{d\left(1+\lambda_{B} \xi(w)\right)}{1+\lambda_{B} \xi(w)},
$$

which gives the solution

$$
F_{2}(w)+i m=c_{2}\left(1+\lambda_{B} \xi(w)\right),
$$

where $c_{2}$ is an integration constant.

In order to determine the constant $c_{2}$ we plug (2.90) into the second of (2.68). Using (2.84) we see that the r.h.s. of that integral equation evaluates to

$$
-c_{2} \lambda_{B} \int_{w}^{\infty} d \xi(w)
$$

The integral (2.91) is divergent and must be evaluated after dimensional regularization. Exactly as in subsubsection 2.9.1, the net result of this regulation scheme is to simply drop the surface term at infinity. We conclude that the integral in (2.91) evaluates to

$$
c_{2} \lambda_{B} \xi(w)
$$

The integral equation is satisfied if this expression also equals $F_{2}$. Using (2.90) this condition takes the form

$$
c_{2}\left(1+\lambda_{B} \xi(w)\right)-i m=c_{2} \lambda_{B} \xi(w) \quad \text { i.e. } \quad c_{2}=i m
$$

We conclude that the unknown function $F_{2}$ is given by

$$
F_{2}(w)=i m \lambda_{B} \xi(w) .
$$

\subsubsection{Determination of the mass}

Plugging (2.93) and (2.79) into (2.70) we find

$$
M^{\prime}(w)=0,
$$

In other words the complicated mass function $M(w)$, listed in (2.54) is just a constant independent of $w$. We pause to recall why this result is extremely satisfying, both from the physical and the technical point of view.

Recall that poles of the $W$ boson lie at the zeroes of the determinant of $Q$. Now the poles of $W$ boson particles have gauge invariant physical content (they determine the dispersion relation of the $W$ bosons). At zero temperature we expect this dispersion to be Lorentz invariant. It follows from (2.54) that this is only the case if $M$ is a constant independent of $w$. The fact that $M$ comes out to be constant and serves as a nontrivial consistency check on our results at zero temperature. ${ }^{22}$

\footnotetext{
${ }^{22}$ Note that the full $W$ boson propagator - which is gauge dependent and so unphysical — is far from Lorentz invariant in our gauge. It is gratifying that, nonetheless, the gauge invariant data in the propagator is Lorentz invariant.
} 
It is not immediately clear that there is a clear physical reason to expect that $M$ had to be constant, independent of $w$, even away from the zero temperature limit. However the fact that this turns out to be the case is satisfying for two reasons. First, it allows us to give a clear interpretation to the quantity $M ; M$ is the 'thermal mass' of the $W$ bosons. More importantly, at the technical level, the fact that $M$ is a constant turns the function $\chi$ into a completely known function of $w$ (it was previously known in terms of the unknown function $M(w)$ ). This fact turns the differential equations for $F_{3}$ and $F_{1}$ into linear differential equations that are easily solved. We will return to this point in the next subsubsection.

Of course the constant value of the mass $M$ is not a free parameter; it is itself determined in terms of the parameters of the theory and the temperature. In the rest of this subsubsection we will find an equation that determines the value of $M$.

Inserting the relation

$$
-\frac{i}{m}\left(F_{2}+i m\right)\left(1-F_{4}\right)=1
$$

into the expression for $M^{2}$ in (2.54) we conclude that

$$
M^{2}=-\left(F_{2}+i m\right)^{2}-\frac{i}{m} F_{1}\left(1-F_{4}\right)^{2}+2 F_{3} .
$$

Eq. (2.96) is a functional relationship that holds at every value of $w$. The r.h.s. of (2.96) involves the functions $F_{3}$ and $F_{1}$ that we still do not know at general values of $w$. However the structure of the last two equations (2.62) ensures that $F_{3}(0)=F_{1}(0)=0$. Evaluating (2.96) at $w=0$ we find the equation

$$
M^{2}=-\left(F_{2}(0)+i m\right)^{2}=m^{2}\left(\lambda_{B} \xi(0)+1\right)^{2} .
$$

Recall

$$
\xi(0)=\frac{1}{2 \beta m} \int d \alpha \rho_{B}(\alpha)\left[\log 2 \sinh \left(\frac{\beta}{2}\left(M+i \frac{\alpha}{\beta}\right)\right)+\log 2 \sinh \left(\frac{\beta}{2}\left(M-i \frac{\alpha}{\beta}\right)\right)\right]=\frac{\mathcal{S}}{m \beta} .
$$

where, we recall from (1.12), that $\mathcal{S}=\mathcal{S}(M, 0)$. To proceed, we define the dimensionless quantities

$$
c_{B}=\beta M, \quad \hat{m}=\beta m .
$$

It follows that $(2.97)$ can be recast in terms of $c_{B}$ and $\hat{m}$ into the equation

$$
c_{B}^{2}=\left(\hat{m}+\frac{\lambda_{B}}{2} \int_{-\pi}^{\pi} d \alpha \rho(\alpha)\left[\log 2 \sinh \left(\frac{c_{B}+i \alpha}{2}\right)+\log 2 \sinh \left(\frac{c_{B}-i \alpha}{2}\right)\right]\right)^{2} .
$$

Using (2.98) and the expression for $\hat{m}$ in terms of $\hat{m}_{B}^{\text {cri }}$ from (A.13), the above may be rewritten as

$$
\left(2 c_{B}\right)^{2}=\left(-\lambda_{B} \hat{m}_{B}^{\text {cri }}+2 \lambda_{B} \mathcal{S}\right)^{2}=\left(-\left|\lambda_{B}\right| \hat{m}_{B}^{\text {cri }}+2\left|\lambda_{B}\right| \mathcal{S}\right)^{2}
$$

This is our final gap equation for the thermal mass of the $W$ bosons. 


\subsubsection{Solving for $F_{3}$ and $F_{1}$}

We next focus on the differential equations for $F_{1}$ and $F_{3}$. We define the function $g(w)$ :

$$
g(w)=\lambda_{B} \xi(w)+1=\frac{1}{i m}\left(F_{2}+i m\right)=\frac{1}{1-F_{4}}, \quad \text { with } \quad g^{\prime}(w)=-\frac{\lambda_{B}}{2(2 \pi)^{3}} \chi(w) .
$$

Then, the differential equation for $F_{3}$ becomes

$$
F_{3}^{\prime}(w)+\frac{g^{\prime}(w)}{g(w)}\left(F_{3}(w)+\frac{w}{2}\right)+m^{2} g^{\prime}(w) g(w)=0,
$$

which we rewrite as

$$
\begin{array}{r}
\left(F_{3}^{\prime}(w)+\frac{1}{2}\right)+\frac{g^{\prime}(w)}{g(w)}\left(F_{3}(w)+\frac{w}{2}\right)+m^{2} g^{\prime}(w) g(w)-\frac{1}{2}=0 \\
\left(\left(F_{3}(w)+\frac{w}{2}\right) g(w)\right)^{\prime}+m^{2} g^{\prime}(w) g(w)^{2}-\frac{1}{2} g(w)=0
\end{array}
$$

Integral equation for $F_{3}$ requires $F_{3}(0)=0$. With this boundary condition above equation can be integrated to give

$$
F_{3}(w)=-\frac{w}{2}+\frac{1}{g(w)}\left(\frac{1}{2} \mathcal{I}(w)-\frac{m^{2}}{3}\left(g(w)^{3}-g(0)^{3}\right)\right)
$$

where we have defined

$$
\mathcal{I}(w)=\int_{0}^{w} g(z) d z
$$

To get a simplified equation for $F_{1}$ we use (2.54) to eliminate $F_{3}$ from r.h.s. of (2.69) ( $M^{2}$ is independent of $w$ as follows from previous discussions) to give a differential equation for $F_{1}$

$$
\begin{aligned}
F_{1}^{\prime}(w)-\frac{g^{\prime}(w)}{g(w)} F_{1}(w)+i m g^{\prime}(w) g(w)\left(g(w)^{2} m^{2}-M^{2}-w\right) & =0 \\
\left(\frac{F_{1}(w)}{g(w)}\right)^{\prime}+i m g^{\prime}(w)\left(g(w)^{2} m^{2}-M^{2}-w\right) & =0
\end{aligned}
$$

Integral equation for $F_{1}$ requires $F_{1}(0)=0$. With this boundary condition above equation can be integrated to give

$$
\begin{aligned}
F_{1}(w) & =i m g(w)\left(M^{2}(g(w)-g(0))-\frac{m^{2}}{3}\left(g(w)^{3}-g(0)^{3}\right)+\int_{0}^{w} z g^{\prime}(z) d z\right) \\
& =i m g(w)\left(M^{2}(g(w)-g(0))-\frac{m^{2}}{3}\left(g(w)^{3}-g(0)^{3}\right)+w g(w)-\mathcal{I}(w)\right)
\end{aligned}
$$

Using formula for mass (2.97), it can be easily checked that these solutions indeed satisfy (2.54) as required by consistency. We next plug these solutions into the effective action and obtain the free energy functional $v_{B}$. Before that, we take a short digression and discuss the case with chemical potential. 


\subsubsection{Adding a chemical potential}

The $\mathrm{SU}\left(N_{B}\right)$ theory (2.1) enjoys invariance under a global $\mathrm{U}(1)$ symmetry. The action of the $\mathrm{U}(1)$ global symmetry element $e^{i \alpha}$ on the fundamental multiplet is given by

$$
\phi \rightarrow e^{i \alpha} U(\alpha) \phi, \quad \bar{\phi} \rightarrow \bar{\phi} U^{\dagger}(\alpha) e^{-i \alpha}, \quad A_{\mu} \rightarrow U(\alpha) A_{\mu} U^{\dagger}(\alpha)
$$

where $U(\alpha)$ is any one parameter choice of $\mathrm{SU}\left(N_{B}\right)$ gauge transformations. As $U(\alpha)$ generate gauge transformations, different choices of $U(\alpha)$ all actually generate the same symmetry. The matrix $U(\alpha)$ can be chosen in any convenient manner.

We will find it convenient to choose $U(\alpha)$ to be given by

$$
U(\alpha)=\operatorname{Diag}\left(e^{i \frac{\alpha}{N_{B}-1}}, e^{i \frac{\alpha}{N_{B}-1}}, \ldots, e^{i \frac{\alpha}{N_{B}-1}}, e^{-i \alpha}\right)
$$

With this choice the action (2.109) preserves our unitary gauge choice (2.5). As we have explained above, this choice of gauge freezes out all $\phi$ degrees of freedom. The gauge fields of the unbroken $\mathrm{SU}\left(N_{B}-1\right)$ gauge group transform trivially under (2.109), as do the neutral $Z$ bosons. On the other hand the fundamental $W$ bosons transform under (2.110) like objects of charge $-\frac{N_{B}}{N_{B}-1}$. In the large $N_{B}$ limit under study in this paper, the charge of these $W$ bosons is just -1 .

The reader may find herself surprised by the fact that the global charge of a $W_{\mu}$ boson differs (although ever so slightly) from -1 . In fact this charge renormalization is actually natural in the $\mathrm{SU}\left(N_{B}\right)$ theory with which we are working. Recall that neither $\phi$ (in the unHiggsed phase) nor $W_{\mu}$ (in the Higgsed phase) are gauge invariant operators. We can build gauge invariant operators by contracting $\phi$ (or $W$ ) with their complex conjugates, but such operators carry no global charge. The simplest charged gauge invariant operator is a 'baryon' build by contracting $N_{B} \phi,{ }^{23}$ or $N_{B}-1 W_{\mu}$ operators. In the unHiggsed phase such a baryon clearly carries global symmetry charge $N_{B}$. This precisely matches the global symmetry charge of a baryon operator made out of $N_{B}-1 \bar{W}_{\mu}$ fields precisely because of the charge 'renormalization' described above. In other words the charge renormalization is precisely what is needed in order to ensure that the charges of gauge invariant operators do not jump as we move from the Higgsed to the unHiggsed phase. ${ }^{24}$

There is another way of understanding fact that the ratio of the magnitude of the charge of a $W$ boson and the original $\phi$ field is $\frac{N_{B}}{N_{B}-1}$ using duality. Let $B_{\mu}$ be the background gauge field that couples to the global $\mathrm{U}(1)$ symmetry of the $\mathrm{U}\left(N_{F}\right)_{k_{F}}$ fermionic theory. The coupling in question is proportional to $\int B d a$ where $a$ is the $\mathrm{U}(1)$ part of the dynamical $\mathrm{U}\left(N_{F}\right)$ fermionic gauge field. A single fermionic particle traps $d a$ flux proportional to $\frac{1}{\left|k_{F}\right|}$ when $m_{F}$ and $k_{F}$ have the same sign (i.e. in the dual of the un Higgsed phase) but $d a$ flux proportional to $\frac{1}{\left|k_{F}\right|-1}$ when $m_{F}$ and $k_{F}$ have opposite signs, i.e. in the dual to of the Higgsed phase. It follows that the ratio of charges of excitations in the (fermionic dual to) the unHiggsed and Higgsed phases is $\frac{\left|k_{F}\right|}{\left|k_{F}\right|-1}$, which exactly maps to $\frac{N_{B}}{N_{B}-1}$ under duality.

\footnotetext{
${ }^{23}$ In our schematic discussion we use the same notation for $\phi$ and any of its derivatives; similarly for $W_{\mu}$.

${ }^{24}$ Note that the phenomenon we have just explained - namely the 'renormalization' of global charge does not occur in the $\mathrm{U}\left(N_{B}\right)$ theory in which case $W_{\mu}$ bosons have global charge -1 . This matches with the fact that the explanation we have presented also does not apply to the $\mathrm{U}\left(N_{B}\right)$ theory which has no baryonic operators.
} 
Let us now repeat the computation of the thermal partition function, presented earlier in this section, after turning on a chemical potential $\mu$ for the $\mathrm{U}(1)$ charge with action listed in (2.109). This may be accomplished by turning on an imaginary background gauge field with $A_{0}=i \mu$ for all fundamental $W$ fields and an imaginary gauge field with $A_{0}=-i \mu$ for the antifundamental $\bar{W}$ fields. This is achieved by making the replacement $\alpha \rightarrow \alpha-i \nu$ in (2.61) and (2.63) etc. In other words we define generalized $\chi$ and $\xi$ functions by the formulae

$$
\begin{aligned}
\chi(z) \equiv & -\frac{8 \pi^{3}}{m} \int d \alpha \rho_{B}(\alpha) \beta^{-1} \sum_{n \in \mathbb{Z}} \frac{1}{\left(2 \pi \frac{n}{\beta}+\frac{\alpha}{\beta}-i \frac{\nu}{\beta}\right)^{2}+\left(z+M^{2}(z)\right)}, \\
= & -\frac{2 \pi^{3}}{m} \int d \alpha \rho_{B}(\alpha) \frac{1}{\sqrt{z+M^{2}}}\left(\operatorname{coth}\left(\frac{\beta}{2}\left(\sqrt{z+M^{2}}+i \frac{\alpha}{\beta}+\frac{\nu}{\beta}\right)\right)\right. \\
& \left.+\operatorname{coth}\left(\frac{\beta}{2}\left(\sqrt{z+M^{2}}-i \frac{\alpha}{\beta}-\frac{\nu}{\beta}\right)\right)\right)
\end{aligned}
$$

and

$$
\begin{aligned}
\xi(z)= & -\frac{1}{2(2 \pi)^{3}} \int^{z} d w^{\prime} \chi\left(w^{\prime}\right), \\
= & \frac{1}{2 \beta m} \int d \alpha \rho_{B}(\alpha)\left(\log 2 \sinh \left(\frac{\beta}{2}\left(\sqrt{z+M^{2}}+i \frac{\alpha}{\beta}+\frac{\nu}{\beta}\right)\right)\right. \\
& \left.+\log 2 \sinh \left(\frac{\beta}{2}\left(\sqrt{z+M^{2}}-i \frac{\alpha}{\beta}-\frac{\nu}{\beta}\right)\right)\right) .
\end{aligned}
$$

Earlier in this subsection we have obtained explicit results for the thermal self energy and propagators of our theory. Our results were expressed in terms of the functions $F_{1} \ldots F_{4}$ which, in turn, we solved for in term of $\chi$ and $\xi$. All of these results also go through in the presence of a chemical potential if we replace the functions $\chi$ and $\xi$ with their generalizations defined in this subsection.

\section{The free energy}

Recall that the free energy functional $v_{B}$ is given in the saddle point approximation by the effective action for the $\alpha$ and $\Sigma$ fields (cf. (2.39)):

$$
\begin{aligned}
\mathcal{V}_{2} T^{2} v_{B}\left(\left|c_{B}\right|, \rho_{B}\right)=S_{\text {eff }}[\alpha, \Sigma]= & N_{B} \mathcal{V}_{3}\left(V_{0}[\alpha]-\frac{i}{\lambda_{B}} \int \frac{\mathcal{D}^{3} q}{(2 \pi)^{3}} \Sigma^{\nu \mu}(q) \alpha_{\mu \nu}(-q)\right. \\
& \left.+\int \frac{\mathcal{D}^{3} q}{(2 \pi)^{3}} \log \operatorname{det}\left(K_{W}(q)-i \Sigma^{T}(q)\right)\right) .
\end{aligned}
$$

The quantity $V_{0}[\alpha]$ in (3.1) is complicated because it involves a double integral over momenta even when evaluated on translationally invariant solutions. On shell, however, it is possible to eliminate $V_{0}[\alpha]$ using the equations of motion. Using the fact that $V_{0}[\alpha]$ is a homogeneous polynomial of degree 2 in $\alpha$ it follows (from the $\alpha$ equation of motion) that, on-shell,

$$
V_{0}[\alpha]=\frac{1}{2} \frac{\delta V_{0}}{\delta \alpha} \cdot \alpha=\frac{1}{2} \frac{i}{\lambda_{B}} \Sigma \cdot \alpha
$$


Plugging this into the equation (3.1) we find that on-shell, the effective action becomes,

$$
S_{\text {eff }}=N_{B} \mathcal{V}_{3}\left(-\frac{i}{2 \lambda_{B}} \int \frac{\mathcal{D}^{3} q}{(2 \pi)^{3}} \Sigma^{\nu \mu}(q) \alpha_{\mu \nu}(-q)+\int \frac{\mathcal{D}^{3} q}{(2 \pi)^{3}} \log \operatorname{det}\left(K_{W}(q)-i \Sigma^{T}(q)\right)\right)
$$

Using the formula (2.54) and the fact that $M(w)=M$ (where $M$ is independent of $w$ ), the second term on the r.h.s. of (3.3) is easily evaluated:

$$
\begin{aligned}
& \int \frac{\mathcal{D}^{3} q}{(2 \pi)^{3}} \log \operatorname{det}\left(K_{W}(q)-i \Sigma^{T}(q)\right) \\
& =-\frac{\left|c_{B}\right|^{3}}{3}+\int_{-\pi}^{\pi} \rho(\alpha) d \alpha \int_{\left|c_{B}\right|}^{\infty} d y y\left(\log \left(1-e^{-y-i \alpha-\nu}\right)+\log \left(1-e^{-y+i \alpha+\nu}\right)\right),
\end{aligned}
$$

(Here and the rest of the analysis in this section, we restrict ourselves to the regime $\left|c_{B}\right|>$ $|\nu|$. See appendix A for a discussion.) We now turn to simplifying the first term on the r.h.s. of (3.3). Using the fact that $\Sigma^{33}=\Sigma^{++}=0$ (see (2.55)) it follows that that

$$
\begin{aligned}
& \Sigma^{\nu \mu}(q) \alpha_{\mu \nu}(-q)= \\
& \quad \Sigma^{--}(q) \alpha_{--}(-q)+2\left(\Sigma^{-3}(q) \alpha_{3-}(-q)+\Sigma^{-+}(q) \alpha_{+-}(-q)+\Sigma^{+3}(q) \alpha_{3+}(-q)\right) .
\end{aligned}
$$

In order to further simplify (3.5) we now plug in the explicit expressions for $\Sigma$ and $\alpha$ obtained above (i.e. (2.55) and (2.56) with the particular values of $F_{1} \ldots F_{4}$ solved for above). The dependence of the resultant expression on $q_{3}$ is very simple; it is given by a polynomial of degree one in $q_{3}$ times $\frac{1}{\operatorname{det} Q}$. (Of course $q_{3}$ is discretized and holonomy shifted version at finite temperature). The linear term in this Polynomial yields a vanishing contribution when summed over the full range of discrete values of $q_{3}$ and simultaneously integrated over the holonomy. ${ }^{25}$ For this reason we simply ignore the term linear in $q_{3}$. With this understanding - omitting the terms discussed above - we have

$$
\begin{aligned}
\Sigma^{\nu \mu}(q) \alpha_{\mu \nu}(-q)= & -\frac{\lambda_{B}}{(2 \pi)^{3} \operatorname{det} Q} \operatorname{im} \mathcal{L}(w) \\
\mathcal{L}(w)= & \frac{2 g(0)^{3} m^{2}+3 \mathcal{I}(w)}{3 g(w)^{2}}+\frac{1}{3}\left(-2 g(0)^{3} m^{2}-9 g(0)^{2} m^{2}-3 \mathcal{I}(w)-6 w\right) \\
& -\frac{4}{3} m^{2} g(w)^{3}+m^{2} g(w)^{2}+\frac{1}{3} g(w)\left(6 g(0)^{2} m^{2}+4 m^{2}+6 w\right)
\end{aligned}
$$

(the functions $g$ and $\mathcal{I}$ were defined in (2.102) and (2.106) above). The dependence of (3.6) on the discretized and holonomy shifted version of $q_{3}$ is entirely through the factor of $\frac{1}{\operatorname{det} Q}$.

\footnotetext{
${ }^{25}$ We use here that the eigenvalue distribution function $\rho(\alpha)$ is an even function of $\alpha$.
} 
Performing the sum over the discrete momenta in $q_{3}$ we find

$$
\begin{aligned}
-\frac{i}{2 \lambda_{B}} \int \frac{\mathcal{D}^{3} q}{(2 \pi)^{3}} \Sigma^{\nu \mu}(q) \alpha_{\mu \nu}(-q) & =\int \frac{\mathcal{D}^{3} q}{(2 \pi)^{3}} \frac{-m}{2(2 \pi)^{3} \operatorname{det} \mathrm{Q}} \mathcal{L}(w) \\
& =\frac{-m}{2(2 \pi)^{3}} \int \frac{q_{s} d q_{s}}{2 \pi} \frac{1}{\beta} \sum_{q_{3}} \frac{1}{-\frac{m}{(2 \pi)^{3}}\left(q^{2}+M^{2}\right)} \mathcal{L}(w) \\
& =\frac{m}{4 \pi} \frac{-1}{2(2 \pi)^{3}} \int d w \chi(w) \mathcal{L}(w)=\frac{m}{4 \pi} \int d w \xi^{\prime}(w) \mathcal{L}(w) \\
& =\frac{m}{4 \pi} \int d w \xi^{\prime}(w) \sum_{n} \mathcal{L}_{n}(w)\left(-\lambda_{B}\right)^{n}
\end{aligned}
$$

where we have used (2.61) and (2.63). In the last line of (3.7) we have simply Taylor expanded $\mathcal{L}$ in all explicit factors of $\lambda_{B}$ according to the following rule. We see from (3.6) that $\mathcal{L}$ depends on the functions $g$ and $\mathcal{I}$. We use the equation (2.102) to rewrite $g$ in terms of $\chi$ using

$$
g(w)=1+\lambda_{B} \xi(w), \quad g^{\prime}(w)=-\frac{\lambda_{B}}{2(2 \pi)^{3}} \chi(w) .
$$

In a similar fashion we use (2.106) to write $\mathcal{I}$ in terms of integrals of $\xi$ :

$$
\mathcal{I}(w)=\int_{0}^{w} d z\left(1+\lambda_{B} \xi(z)\right) .
$$

We then Taylor expand $\mathcal{L}$ treating $\xi$ and all its integrals as independent of $\lambda_{B}$; with this understanding $\mathcal{L}_{n}$ are defined by

$$
\mathcal{L}=\sum_{n=0}^{\infty} \mathcal{L}_{n}(w)\left(-\lambda_{B}\right)^{n} .
$$

The various coefficient functions $\mathcal{L}_{n}$ are easily worked out. We find ${ }^{26}$

$$
\begin{aligned}
\mathcal{L}_{0}(w)= & 0, \quad \mathcal{L}_{1}(w)=2 m^{2} \xi[0] \\
\mathcal{L}_{2}(w)= & -2 \mathcal{I}_{\xi}(w) \xi(w)-m^{2} \xi(0)^{2}-m^{2} \xi(w)^{2}+3 w \xi(w)^{2} \\
\mathcal{L}_{3}(w)= & -3 \mathcal{I}_{\xi}(w) \xi(w)^{2}+4 m^{2} \xi(w)^{3}-6 m^{2} \xi(0) \xi(w)^{2}+2 m^{2} \xi(0)^{2} \xi(w)+4 w \xi(w)^{3} \\
\mathcal{L}_{n}(w)= & \frac{1}{3} \xi(w)^{n-3}\left(-6 n \xi(w)^{2} m^{2} \xi(0)-2 m^{2}(n-2) \xi(0)^{3}+6 m^{2}(n-1) \xi(0)^{2} \xi(w)\right. \\
& \left.+(n+1) 2 m^{2} \xi(w)^{3}\right)+\left((n+1) w \xi(w)^{n}-n \xi(w)^{n-1} \mathcal{I}_{\xi}(w)\right) \quad \text { for } \quad n \geq 4
\end{aligned}
$$

where

$$
\mathcal{I}_{\xi}(w)=\int_{0}^{w} \xi(z) d z
$$

The integral (3.7) over the last two terms in the expression for $\mathcal{L}_{n}, n \geq 4$ in (3.11) can be simplified using

$$
d w \xi^{\prime}(w)\left((n+1) \xi(w)^{n} w-n \xi(w)^{n-1} \mathcal{I}_{\xi}(w)\right)=d\left(\xi(w)^{n+1} w-\xi(w)^{n} \mathcal{I}_{\xi}(w)\right)
$$

\footnotetext{
${ }^{26} \mathcal{L}_{0}(w)=0$ is just the statement that this contribution is present only when interactions with gauge fields are turned on.
} 
It follows that the integral over those terms reduces to surface terms which vanish in the dimensional regularization scheme ${ }^{27}$ so that

$$
\int d w \xi^{\prime}(w)\left((n+1) \xi(w)^{n} w-n \xi(w)^{n-1} \mathcal{I}_{\xi}(w)\right)=0 .
$$

The integral over all remaining terms in $\mathcal{L}_{n}$ for all $n$ are of the form

$$
\int d \xi f(\xi)
$$

where the functions $f$ are all simple polynomials of $\xi$. As a consequence all remaining integrals are easily performed and we find

$$
\int d w \xi^{\prime}(w) \mathcal{L}_{n}(w)=0 \quad \text { for } n \geq 3
$$

The only non-zero contributions are

$$
\begin{aligned}
& \int d w \xi^{\prime}(w) \mathcal{L}_{1}(w)=-2 m^{2} \xi[0]^{2} \\
& \int d w \xi^{\prime}(w) \mathcal{L}_{2}(w)=\frac{4}{3} m^{2} \xi[0]^{3} .
\end{aligned}
$$

Putting all these together we get, for the $\Sigma \cdot \alpha$ piece,

$$
\begin{aligned}
-N_{B} \mathcal{V}_{3} \frac{i}{2 \lambda_{B}} \int \Sigma^{\nu \mu}(q) \alpha_{\mu \nu}(-q) & =N_{B} \frac{m \mathcal{V}_{3}}{4 \pi}\left(2 m^{2} \xi[0]^{2} \lambda_{B}+\frac{4}{3} m^{2} \xi[0]^{3} \lambda_{B}^{2}\right) \\
& =N_{B} \frac{\mathcal{V}_{2} T^{2}}{6 \pi}\left(3 \mathcal{S}^{2}|\hat{m}|\left|\lambda_{B}\right|+2 \mathcal{S}^{3} \lambda_{B}^{2}\right)
\end{aligned}
$$

where we use $\operatorname{sgn}(m)=\operatorname{sgn}\left(\kappa_{B}\right)=\operatorname{sgn}\left(\lambda_{B}\right)$. Combining (3.18) and (3.4) we obtain

$$
\begin{aligned}
v_{B}\left(\left|c_{B}\right|, \rho_{B}\right)= & \frac{N_{B}}{6 \pi}\left(3\left|\lambda_{B}\right||\hat{m}| \mathcal{S}^{2}+2\left|\lambda_{B}\right|^{2} \mathcal{S}^{3}\right. \\
& -\left|c_{B}\right|^{3}+3 \int_{-\pi}^{\pi} \rho_{B}(\alpha) d \alpha \int_{\left|c_{B}\right|}^{\infty} d y y\left(\log \left(1-e^{-y-i \alpha-\nu}\right)+\log \left(1-e^{-y+i \alpha+\nu}\right)\right)
\end{aligned}
$$

This matches precisely with the prediction for the bosonic free energy from the fermionic result presented in (1.19). In other words the free energy of the bosonic theory exactly matches the free energy of the fermionic theory under the duality map, as we set out to show.

It is not difficult to promote the expression (3.19) to an offshell free energy. Consider the quantity

$$
\begin{aligned}
F_{B}\left[\rho_{B}(\alpha), c_{B}\right]= & \frac{N_{B}}{6 \pi}\left[-\frac{\left(\lambda_{B}-\operatorname{sgn}\left(\lambda_{B}\right)-\operatorname{sgn}\left(X_{B}\right)\right)}{\lambda_{B}}\left|c_{B}\right|^{3}+\frac{3}{2} \hat{m}_{B}^{\text {cri }} c_{B}^{2}+\alpha\left(\hat{m}_{B}^{\text {cri }}\right)^{3}\right. \\
& \left.+3 \int_{-\pi}^{\pi} \rho(\alpha) d \alpha \int_{\left|c_{B}\right|}^{\infty} d y y\left(\ln \left(1-e^{-y-i \alpha}\right)+\ln \left(1-e^{-y+i \alpha}\right)\right)\right],
\end{aligned}
$$

\footnotetext{
${ }^{27}$ The fact that $\left.\xi(\infty)\right|_{D R}=0, \mathcal{I}_{\xi}(0)=0$ is used here.
} 
where $\alpha$ is an unknown pure number (see below for a discussion). In the case that $\operatorname{sgn}\left(X_{B}\right)=-\operatorname{sgn}\left(\lambda_{B}\right), F_{B}$ reduces to $v_{B}(\rho)$ reported in (1.10). It follows that (3.20) is the correct offshell free energy in the unHiggsed phase. Let us now consider the opposite case $\operatorname{sgn}\left(X_{B}\right)=\operatorname{sgn}\left(\lambda_{B}\right)$. In this case the expression for $F_{B}$ in (3.20) simplifies to

$$
\begin{aligned}
F_{B}\left[\rho_{B}(\alpha), c_{B}\right]= & \frac{N_{B}}{6 \pi}\left[-\frac{\left(\lambda_{B}-2 \operatorname{sgn}\left(\lambda_{B}\right)\right)}{\lambda_{B}}\left|c_{B}\right|^{3}+\frac{3}{2} \hat{m}_{B}^{\text {cri }} c_{B}^{2}+\alpha\left(\hat{m}_{B}^{\text {cri }}\right)^{3}\right. \\
& \left.+3 \int_{-\pi}^{\pi} \rho(\alpha) d \alpha \int_{\left|c_{B}\right|}^{\infty} d y y\left(\ln \left(1-e^{-y-i \alpha}\right)+\ln \left(1-e^{-y+i \alpha}\right)\right)\right]
\end{aligned}
$$

It is not difficult to verify that the condition of stationarity of variation of (3.21) w.r.t. $\left|c_{B}\right|$ yields the gap equation (2.101). It is also not difficult to verify that when (3.21) reduces to (3.19) when evaluated onshell (upto the term proportional to $\alpha$ : see below) i.e. on a solution to (3.21). It follows that (3.21) is an offshell free energy for the critical boson theory in the Higgsed phase, and so also that (3.20) is an offshell free energy for the critical boson in either phase - Higgsed or unHiggsed. Finally, it is not difficult to verify that (3.20) maps to the regular fermionic offshell free energy reported in (1.10) (once we identify $\left|c_{B}\right|$ with $\left.\left|c_{F}\right|\right)$.

Let us now return to a discussion of the parameter $\alpha$ in (3.21). As this term is independent of $c_{B}$ it does not affect the variation of the action w.r.t. $c_{B}$ and so does not contribute to the gap equations. This term shifts $\ln Z$ of the theory $(Z$ is the finite temperature partition function) by $-\frac{V\left(m_{B}^{\text {cri }}\right)^{3} \alpha}{T}$ where $V$ is the volume of space and $T$ is the temperature. This shift can be absorbed into a shift of the ground state energy of the theory by $V \alpha\left(m_{B}^{\text {cri }}\right)^{3}$, or equivalently by a shift proportional to $\alpha\left(m_{B}^{\text {cri }}\right)^{3}$ of the cosmological constant counterterm of the original field theory. In other words the parameter $\alpha$ can only be determined once we have made a particular choice of the cosmological constant counterterm. In the absence of such a choice $\alpha$ is ambiguous. We will leave $\alpha$ above as a free parameter in our final result.

As we have explained in the introduction, the quantity $v_{B}$ reported in (3.19) (or equivalently (1.19)) defines the integrand of an integral over unitary matrices $U$. The result of this integral over $U$ is the finite temperature partition function $\mathcal{Z}$

$$
\mathcal{Z}=\operatorname{Tr} e^{-\beta H},
$$

where $H$ is the Hamiltonian. In the Higgsed phase the Hamiltonian $H$ may be obtained by canonically quantizing the action (2.7) - the starting point of our path integral evaluation of the free energy. The spectrum of (2.7) is particularly simple in the limit $\lambda_{B}=0$ with $\left|m_{W}\right|=\left|\frac{\lambda_{B} m_{B}^{\text {cri }}}{2}\right|$ held fixed. In this limit the gauge fields $A_{\mu}$ are very weakly coupled, and the the partition function (3.22) may be evaluated by enumerating the spectrum of effectively free massive $W$ (and $Z$ ) bosons, subject only to the 'Gauss Law' constraint that asserts that all physical states are gauge singlets (see [61] and references therein). It is easy to see that our explicit results (1.17) and (1.19) are consistent with this expectation. In this limit (1.17) reduces to $\left|c_{B}\right|=\left|\hat{m}_{W}\right|$. In other words the thermal mass of the $W$ bosons 
agrees with their bare mass at all temperatures, as expected in a free theory. Moreover, after dropping irrelevant constants, the expression (1.19) reduces, in this limit to

$$
v_{B}\left(\left|c_{B}\right|, \rho_{B}\right)=\frac{N_{B}}{2 \pi} \int_{-\pi}^{\pi} \rho_{B}(\alpha) d \alpha \int_{\left|\hat{m}_{W}\right|}^{\infty} d y y\left(\log \left(1+e^{-y-i \alpha-\nu}\right)+\log \left(1+e^{-y+i \alpha+\nu}\right)\right),
$$

which is precisely $v_{B}$ of a free complex bosonic degree of freedom (in this case the $W$ bosons) in the fundamental representation. ${ }^{28}$

\section{Discussion}

In this paper we have directly evaluated the thermal free energy of the large $N_{B}$ ChernSimons gauged critical scalar theory in its Higgsed phase, and demonstrated that our final results match perfectly with the predictions of its conjectured fermionic dual. In particular we have demonstrated that the pole mass of the $W$ boson maps to the pole mass of the bare fermionic excitations under duality. It follows that under duality, the elementary fermionic excitations - which map to elementary scalar excitations in the unHiggsed phase map to $W$ bosons in the Higgsed phase.

At zero temperature both the bosonic theory and its fermionic dual undergo a sharp phase transition when the bosonic/fermionic mass goes through zero. As we have explained above, the topological pure Chern-Simons theory that governs the long distance dynamics of the two theories changes discontinuously from positive to negative mass, and may be thought of as an order parameter for the phase transition. At finite temperature, on the other hand, there is no clear order parameter separating the two 'phases' (note in particular that the long distance effective theory is two rather than three dimensional and so cannot be a Chern-Simons theory). On physical grounds it seems likely that the free energy of our theories is analytic as a function of mass (and chemical potentials) even at finite $N$.

It is interesting that this physically expected feature of the free energy - namely that it is analytic as a function of mass at finite temperature - is borne out by the explicit large $N_{B}$ calculations presented in this paper but in a highly unusual way. The finite temperature free energy of the bosonic theory in its Higgsed 'phase' is determined by a completely different computation than the one that determines the finite temperature free energy in the unHiggsed 'phase'. The two calculations have non overlapping domains of validity, deal with different degrees of freedom and are dominated by distinct looking saddle points. Yet, when the dust settles, it turns out (in an apparently miraculous manner) that the two results are simply analytic continuations of each other. At the level of formulas, therefore, there is a sense in which the duality between fermions and scalars is enhanced into a 'triality' between fermions, scalars and $W$ bosons at finite temperature: there are three completely different looking computations, each of which give rise to the same final free energy after the appropriate analytic continuation. It would be interesting to understand this better - perhaps there is a more general uniform way of computing the bosonic free energy in both phases at once which makes the analyticity of the final result manifest.

\footnotetext{
${ }^{28}$ We thank D. Radicevic for a very useful discussion on this point.
} 
From a physical point of view, the duality between bosons and fermions is particularly interesting at nonzero chemical potential and low temperatures. In this regime one expects a Fermi liquid at weak fermionic coupling but a Bose condensate at weak bosonic coupling. By analyzing the already known fermionic results, the authors of [25] have already made this expectation quantitative (by dualizing the fermionic free energy to bosonic variables, and demonstrating that the final results at weak bosonic coupling enjoys certain features expected of Bose condensates). It would be interesting to better understand these results directly from the bosonic point of view using the results of this paper.

The partition function of the Higgsed phase scalar theory on $S^{2} \times S^{1}$ is obtained by performing an integral over holonomies; the integrand for this integral is given by the the free energy $v_{B}[\rho]$ computed in this paper. From a physical point of view it would be interesting to explore this integral in detail, particularly at finite chemical potential. In the large volume limit the saddle point eigenvalue distribution will take the universal tabletop form. ${ }^{29}$ However the distribution will deviate from this universal form away from the large volume limit, giving rise to a rich phase structure with many interesting phase transitions (generalizing the analysis of [19]).

It should be possible to generalize the computations presented in this paper to the study of the partition functions of the regular boson - critical fermion duality (see e.g. [23]) and of theories with with both a bosonic and a fermionic field $([22,24])$. It would also be interesting to use the techniques of this paper to generalize the S-matrix computations of [26-31] to evaluate the bosonic S-matrices in the Higgsed phase, and to match the final results with the fermionic S-matrices as predicted by duality. The techniques of this paper could also permit the computation of the quantum effective action of the scalar theories as a function of the gauge covariant field $\phi^{a}$ (in a suitable gauge). This computation could prove useful in analysing the vacuum stability of these theories. We hope to turn to several of these issues in the near future.

\section{Acknowledgments}

We would like to thank T. Sharma, T. Takimi, S. Wadia and S. Yokoyama for collaboration during the initial stages of this project. We would also like to thank O. Aharony, A. Gadde, D. Radicevic, and D. T. Son for useful discussions, and O. Aharony, D. Radicevic, S. Prakash, N. Seiberg and S. Wadia for useful comments on the manuscript. The work of S. C., A. D., I. H., L. J., S. M., and N. P. was supported by the Infosys Endowment for the study of the Quantum Structure of Spacetime. S. C. and S. J. would like to thank TIFR, Mumbai for hospitality during the completion of the work. Finally we would all like to acknowledge our debt to the steady support of the people of India for research in the basic sciences.

\footnotetext{
${ }^{29}$ Given by $\rho(\alpha)=0$ for $|\alpha|>\pi\left|\lambda_{B}\right|$ and $\rho(\alpha)=\frac{1}{2 \pi\left|\lambda_{B}\right|}$ for $|\alpha|<\pi\left|\lambda_{B}\right|$.
} 


\section{A Review of known results and a prediction for the Higgsed phase}

The gap equation for the bosonic theory — which follows from varying (1.10) w.r.t. $\left|c_{B}\right|$ - takes the form

$$
2 \mathcal{S}\left(\left|c_{B}\right|, \nu\right)=\hat{m}_{B}^{\text {cri }},
$$

while the gap equation for the fermionic theory is

$$
\left|c_{F}\right|=\operatorname{sgn}\left(X_{F}\right)\left(2 \lambda_{F} \mathcal{C}\left(\left|c_{F}\right|, \nu\right)+\hat{m}_{F}^{\mathrm{reg}}\right)=\left|X_{F}\right|,
$$

where $\mathcal{S}$ and $\mathcal{C}$ were defined in (1.12).

The bosonic and fermionic holonomy eigenvalue distribution functions are related to each other by the formula (see [19])

$$
\left|\lambda_{B}\right| \rho_{B}(\alpha)+\left|\lambda_{F}\right| \rho_{F}(\pi-\alpha)=\frac{1}{2 \pi} .
$$

When (A.3) holds (and assuming that $\rho_{B}(\alpha)$ and $\rho_{F}(\alpha)$ are even functions of their arguments) it is easily verified that

$$
\begin{aligned}
& \lambda_{B} \mathcal{S}=\lambda_{F} \mathcal{C}-\frac{\operatorname{sgn}\left(\lambda_{F}\right)}{2} \max \left(\left|c_{F}\right|,|\nu|\right), \\
& \lambda_{F} \mathcal{C}=\lambda_{B} \mathcal{S}-\frac{\operatorname{sgn}\left(\lambda_{B}\right)}{2} \max \left(\left|c_{B}\right|,|\nu|\right) .
\end{aligned}
$$

The equations (A.3) have been derived assuming that the integral over $\alpha$ in the first of (A.4) runs over real $\alpha$, i.e. the unit circle in the complex plane $z=e^{i \alpha}$.

We pause to elaborate on the analytic structure of the functions $\mathcal{C}$ and $\mathcal{S}$. The integrals over $\alpha$ in $\mathcal{C}$ and $\mathcal{S}$ formally run over the range $(-\pi, \pi)$. In this paper we will, however, be mainly interested in phases in which $\rho_{F}(\alpha)$ vanishes in a neighbourhood of $\pi$ (see [19]) for an extensive discussion of the phases of the large $N$ partition functions of this theory). In the rest of this paragraph we focus our attention on these fermionic 'lower gap' phases. When this is the case, it is easy to check that the argument of the logarithmic functions that appear in $\mathcal{C}\left(\left|c_{F}\right|, \nu\right)$ in (1.12) never pass through either zero or any negative number for any value of $\left|c_{B}\right|$ or $\nu$. It follows that $\mathcal{C}\left(\left|c_{F}\right|, \nu\right)$ is an analytic function of its arguments for all values of $\left|c_{F}\right|$ and $\nu$.

The arguments of $\mathcal{S}\left(\left|c_{B}\right|, \nu\right)$ in (1.12) are also nowhere negative on the (unit circle) contour of integration when $\left|c_{B}\right|>|\nu|$. It follows that $\mathcal{S}$ is also an analytic function of $\nu$ for $\left|c_{B}\right|>|\nu|$. At $\left|c_{B}\right|=\nu$, on the other hand, the arguments of one of the two logarithms in this equation goes to zero at $\alpha=0$. For $|\nu|>\left|c_{B}\right|$, the contour integral passes through the cut of the logarithm. These observations suggest that $\mathcal{S}\left(\left|c_{B}\right|, \nu\right)$ - viewed as a function of $\nu$ at fixed $\left|c_{B}\right|$ - might well be non-analytic at $\nu= \pm\left|c_{B}\right|$. Equation (A.4) - together with the fact that $\mathcal{C}$ is analytic at $\nu= \pm\left|c_{B}\right|$ - tells us that this is indeed the case. Indeed the function $\mathcal{S}$ must have precisely the singularity needed to cancel that of the function $\frac{\operatorname{sgn}\left(\lambda_{B}\right)}{2} \max \left(\left|c_{B}\right|,|\nu|\right)$ on the r.h.s. of the second of (A.4).

The discussion of the last paragraph motivates us to define the analytic function $\tilde{\mathcal{S}}$

$$
\tilde{\mathcal{S}}=\left\{\begin{array}{ccc}
\mathcal{S} & \text { when } & |\nu|<\left|c_{B}\right| \\
\mathcal{S}-\frac{1}{2\left|\lambda_{B}\right|}\left(|\nu|-\left|c_{B}\right|\right) & \text { when } & |\nu|>\left|c_{B}\right|
\end{array} .\right.
$$


When expressed in terms of $\tilde{\mathcal{S}}$ the relations between $\mathcal{S}$ and $\mathcal{C}$ in (A.4) become the single relation

$$
\lambda_{F} \mathcal{C}=\lambda_{B} \tilde{\mathcal{S}}-\frac{\operatorname{sgn}\left(\lambda_{B}\right)}{2}\left|c_{B}\right|
$$

Roughly speaking $\tilde{\mathcal{S}}$ can be thought of as being defined by the same integral as that for $\mathcal{S}$ in the second of (1.12) except that one is instructed to perform the integral over a contour that is deformed to avoid cutting the branch cut of the logarithmic functions.

It follows from (A.6) that under duality the quantity $X_{F}=2 \lambda_{F} \mathcal{C}+\hat{m}_{F}^{\text {reg }}$ defined in (1.11) maps to $X_{B}$ where

$$
X_{B}=2 \lambda_{B} \tilde{\mathcal{S}}-\lambda_{B} \hat{m}_{B}^{\text {cri }}-\operatorname{sgn}\left(\lambda_{B}\right)\left|c_{B}\right| .
$$

Notice that on-shell (i.e. on a solution to the bosonic gap equations)

$$
X_{B}=-\operatorname{sgn}\left(\lambda_{B}\right) \max \left(\left|c_{B}\right|,|\nu|\right), \quad \text { so that }-\lambda_{B} X_{B} \geq 0 .
$$

In other words all solutions to the bosonic gap equations have $\lambda_{B} X_{B} \leq 0$ i.e. $\lambda_{F} X_{F} \geq 0$. It follows that any solution of the fermionic gap equations that violates this inequality does not have a bosonic dual. We will now see how this works in more detail.

Inserting (A.6) into the fermionic gap equation (A.2) we obtain

$$
\left|c_{B}\right|=\operatorname{sgn}\left(X_{B}\right)\left(2 \lambda_{B} \tilde{\mathcal{S}}-\operatorname{sgn}\left(\lambda_{B}\right)\left|c_{B}\right|-\lambda_{B} \hat{m}_{B}^{\mathrm{cri}}\right),
$$

Equivalently

$$
\left|c_{B}\right|\left(1+\operatorname{sgn}\left(\lambda_{B}\right) \operatorname{sgn}\left(X_{B}\right)\right)=\operatorname{sgn}\left(X_{B}\right)\left(2 \lambda_{B} \tilde{\mathcal{S}}-\lambda_{B} \hat{m}_{B}^{\text {cri }}\right),
$$

Let us first suppose that $\operatorname{sgn}\left(\lambda_{B}\right) \operatorname{sgn}\left(X_{B}\right)=-1$. In this case (A.10) reduces to the equation

$$
2 \tilde{\mathcal{S}}=\hat{m}_{B}^{\text {cri }}
$$

This equation matches perfectly with (A.1) when $\left|c_{B}\right|>|\nu| \cdot{ }^{30}$ On the other hand when $\operatorname{sgn}\left(\lambda_{B}\right) \operatorname{sgn}\left(X_{B}\right)=+1,($ A.10) becomes

$$
2\left|c_{B}\right|=\left(2\left|\lambda_{B}\right| \tilde{\mathcal{S}}-\left|\lambda_{B}\right| \hat{m}_{B}^{\mathrm{cri}}\right) .
$$

This is a completely new bosonic gap equation that - at least superficially — seems different from the bosonic gap equation (A.1). It has been speculated that this equations governs the dynamics of the critical boson theory in its Higgsed phase. In the rest of this paper we demonstrate that this is indeed the case by directly deriving (A.12) from an analysis of the bosonic theory.

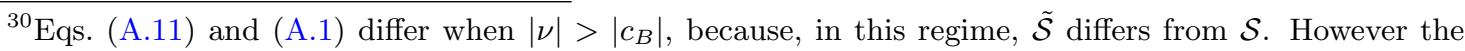
difference between the two equations is quite minor — as we have explained above $\tilde{\mathcal{S}}$ and $\mathcal{S}$ are defined by the same integrals but over slightly different contours. It is possible that the derivation of (A.1) has a subtlety when $|\nu|>\left|c_{B}\right|$ and the correct equation picks out the contour that changes $\mathcal{S}$ to $\tilde{\mathcal{S}}$. We leave an exploration of this to future work.
} 
We can also use the fermionic free energy (the second of (1.10)) together with the duality map to obtain a prediction for the free energy in Higgsed phase (we focus on the case for boson for $\left|c_{B}\right|>|\nu|$; when $\left|c_{B}\right|<|\nu|$ there is a potential subtlety as in the unHiggsed phase).

For later use we present our results in terms of a quantity

$$
m=-\frac{\lambda_{B} m_{B}^{\mathrm{cri}}}{2} \Longrightarrow|m|=-\frac{\left|\lambda_{B}\right| m_{B}^{\mathrm{cri}}}{2}
$$

(and correspondingly for the dimensionless hatted quantities.) Note that in the phase under consideration $m_{B}^{\text {cri }}<0$. The quantity $|m|$ would then correspond to the mass of the $W$ boson in this phase. Using (A.12) we find

$$
\left|c_{B}\right|=\left|\lambda_{B}\right| \mathcal{S}+|\hat{m}|
$$

Substituting (A.14) in (1.10), we have (dropping zero temperature contributions)

$$
\begin{aligned}
v_{F}= & \frac{N_{F}}{6 \pi}\left[\left|c_{F}\right|^{3} \frac{\left(\left|\lambda_{F}\right|+1\right)}{\left|\lambda_{F}\right|}-\frac{3}{2\left|\lambda_{F}\right|}\left|\hat{m}_{F}^{\mathrm{reg}}\right| c_{F}^{2}-\right. \\
& \left.-3 \int_{-\pi}^{\pi} \rho_{F}(\alpha) d \alpha \int_{\left|c_{F}\right|}^{\infty} d y y\left(\log \left(1+e^{-y-i \alpha-\nu}\right)+\log \left(1+e^{-y+i \alpha+\nu}\right)\right)\right] \\
= & \frac{N_{B}}{6 \pi}\left[\left|c_{B}\right|^{3} \frac{\left(2-\left|\lambda_{B}\right|\right)}{\left|\lambda_{B}\right|}-\frac{3}{\left|\lambda_{B}\right|}|\hat{m}| c_{B}^{2}+\right. \\
& \left.+3 \int_{-\pi}^{\pi} \rho_{B}(\alpha) d \alpha \int_{\left|c_{B}\right|}^{\infty} d y y\left(\log \left(1+e^{-y-i \alpha-\nu}\right)+\log \left(1+e^{-y+i \alpha+\nu}\right)\right)\right] \\
= & \frac{N_{B}}{6 \pi}\left[\frac{2\left|c_{B}\right|^{3}-3|\hat{m}| c_{B}^{2}}{\left|\lambda_{B}\right|}+\right. \\
& \left.-\left|c_{B}\right|^{3}+3 \int_{-\pi}^{\pi} \rho_{B}(\alpha) d \alpha \int_{\left|c_{B}\right|}^{\infty} d y y\left(\log \left(1+e^{-y-i \alpha-\nu}\right)+\log \left(1+e^{-y+i \alpha+\nu}\right)\right)\right] \\
= & \frac{N_{B}}{6 \pi}\left[-\frac{|\hat{m}|^{3}}{\left|\lambda_{B}\right|}+3\left|\lambda_{B}\right||\hat{m}| \mathcal{S}^{2}+2\left|\lambda_{B}\right|^{2} \mathcal{S}^{3}\right. \\
& \left.+3 \int_{-\pi}^{\pi} \rho_{B}(\alpha) d \alpha \int_{\left|c_{B}\right|}^{\infty} d y y\left(\log \left(1+e^{-y-i \alpha-\nu}\right)+\log \left(1+e^{-y+i \alpha+\nu}\right)\right)-\left|c_{B}\right|^{3}\right]
\end{aligned}
$$

where we have used the following duality maps in the first step:

$$
\hat{m}_{F}^{\mathrm{reg}}=2 \hat{m}, \quad \frac{N_{F}}{\left|\lambda_{F}\right|}=\frac{N_{B}}{\left|\lambda_{B}\right|}, \quad\left|\lambda_{F}\right|=1-\left|\lambda_{B}\right|, \quad\left|\lambda_{F}\right| \rho_{F}(\alpha)=\frac{1}{2 \pi}-\left|\lambda_{B}\right| \rho(\pi-\alpha) .
$$

In the second and third steps, we have rearranged terms in the expression in order to put it in a form which will match term by term with the free energy obtained by direct calculation in the Higgsed phase. The first term in the bracket is a zero temperature contribution and can be ignored in this context. 
Open Access. This article is distributed under the terms of the Creative Commons Attribution License (CC-BY 4.0), which permits any use, distribution and reproduction in any medium, provided the original author(s) and source are credited.

\section{References}

[1] S. Giombi, S. Minwalla, S. Prakash, S.P. Trivedi, S.R. Wadia and X. Yin, Chern-Simons Theory with Vector Fermion Matter, Eur. Phys. J. C 72 (2012) 2112 [arXiv:1110.4386] [INSPIRE].

[2] I.R. Klebanov and A.M. Polyakov, AdS dual of the critical $O(N)$ vector model, Phys. Lett. B 550 (2002) 213 [hep-th/0210114] [INSPIRE].

[3] E. Sezgin and P. Sundell, Massless higher spins and holography, Nucl. Phys. B 644 (2002) 303 [Erratum ibid. B 660 (2003) 403] [hep-th/0205131] [INSPIRE].

[4] S. Giombi and X. Yin, Higher Spin Gauge Theory and Holography: The Three-Point Functions, JHEP 09 (2010) 115 [arXiv:0912.3462] [INSPIRE].

[5] C.-M. Chang, S. Minwalla, T. Sharma and X. Yin, ABJ Triality: from Higher Spin Fields to Strings, J. Phys. A 46 (2013) 214009 [arXiv:1207.4485] [InSPIRE].

[6] J. Maldacena and A. Zhiboedov, Constraining Conformal Field Theories with A Higher Spin Symmetry, J. Phys. A 46 (2013) 214011 [arXiv:1112.1016] [INSPIRE].

[7] J. Maldacena and A. Zhiboedov, Constraining conformal field theories with a slightly broken higher spin symmetry, Class. Quant. Grav. 30 (2013) 104003 [arXiv:1204.3882] [INSPIRE].

[8] O. Aharony, G. Gur-Ari and R. Yacoby, Correlation Functions of Large N Chern-Simons-Matter Theories and Bosonization in Three Dimensions, JHEP 12 (2012) 028 [arXiv: 1207.4593] [INSPIRE].

[9] G. Gur-Ari and R. Yacoby, Correlators of Large N Fermionic Chern-Simons Vector Models, JHEP 02 (2013) 150 [arXiv: 1211.1866] [INSPIRE].

[10] A. Bedhotiya and S. Prakash, A test of bosonization at the level of four-point functions in Chern-Simons vector models, JHEP 12 (2015) 032 [arXiv:1506.05412] [INSPIRE].

[11] G.J. Turiaci and A. Zhiboedov, Veneziano Amplitude of Vasiliev Theory, JHEP 10 (2018) 034 [arXiv: 1802.04390] [INSPIRE].

[12] O. Aharony, Baryons, monopoles and dualities in Chern-Simons-matter theories, JHEP 02 (2016) 093 [arXiv: 1512.00161] [INSPIRE].

[13] N. Seiberg, T. Senthil, C. Wang and E. Witten, A Duality Web in $2+1$ Dimensions and Condensed Matter Physics, Annals Phys. 374 (2016) 395 [arXiv:1606.01989] [InSPIRE].

[14] A. Karch and D. Tong, Particle-Vortex Duality from 3d Bosonization, Phys. Rev. X 6 (2016) 031043 [arXiv: 1606.01893] [INSPIRE].

[15] J. Murugan and H. Nastase, Particle-vortex duality in topological insulators and superconductors, JHEP 05 (2017) 159 [arXiv: 1606.01912] [INSPIRE].

[16] S. Jain, S.P. Trivedi, S.R. Wadia and S. Yokoyama, Supersymmetric Chern-Simons Theories with Vector Matter, JHEP 10 (2012) 194 [arXiv:1207.4750] [INSPIRE].

[17] S. Yokoyama, Chern-Simons-Fermion Vector Model with Chemical Potential, JHEP 01 (2013) 052 [arXiv:1210.4109] [INSPIRE]. 
[18] O. Aharony, S. Giombi, G. Gur-Ari, J. Maldacena and R. Yacoby, The Thermal Free Energy in Large N Chern-Simons-Matter Theories, JHEP 03 (2013) 121 [arXiv:1211.4843] [INSPIRE].

[19] S. Jain, S. Minwalla, T. Sharma, T. Takimi, S.R. Wadia and S. Yokoyama, Phases of large N vector Chern-Simons theories on $S^{2} \times S^{1}$, JHEP 09 (2013) 009 [arXiv:1301.6169] [INSPIRE].

[20] T. Takimi, Duality and higher temperature phases of large N Chern-Simons matter theories on $S^{2} \times S^{1}$, JHEP 07 (2013) 177 [arXiv: 1304.3725] [INSPIRE].

[21] S. Yokoyama, A Note on Large N Thermal Free Energy in Supersymmetric Chern-Simons Vector Models, JHEP 01 (2014) 148 [arXiv:1310.0902] [INSPIRE].

[22] S. Jain, S. Minwalla and S. Yokoyama, Chern Simons duality with a fundamental boson and fermion, JHEP 11 (2013) 037 [arXiv:1305.7235] [INSPIRE].

[23] S. Minwalla and S. Yokoyama, Chern Simons Bosonization along RG Flows, JHEP 02 (2016) 103 [arXiv:1507.04546] [INSPIRE].

[24] G. Gur-Ari and R. Yacoby, Three Dimensional Bosonization From Supersymmetry, JHEP 11 (2015) 013 [arXiv:1507.04378] [INSPIRE].

[25] M. Geracie, M. Goykhman and D.T. Son, Dense Chern-Simons Matter with Fermions at Large N, JHEP 04 (2016) 103 [arXiv: 1511.04772] [INSPIRE].

[26] S. Jain, M. Mandlik, S. Minwalla, T. Takimi, S.R. Wadia and S. Yokoyama, Unitarity, Crossing Symmetry and Duality of the S-matrix in large N Chern-Simons theories with fundamental matter, JHEP 04 (2015) 129 [arXiv:1404.6373] [INSPIRE].

[27] Y. Dandekar, M. Mandlik and S. Minwalla, Poles in the S-Matrix of Relativistic Chern-Simons Matter theories from Quantum Mechanics, JHEP 04 (2015) 102 [arXiv:1407.1322] [INSPIRE].

[28] K. Inbasekar, S. Jain, S. Mazumdar, S. Minwalla, V. Umesh and S. Yokoyama, Unitarity, crossing symmetry and duality in the scattering of $\mathcal{N}=1$ SUSY matter Chern-Simons theories, JHEP 10 (2015) 176 [arXiv:1505.06571] [INSPIRE].

[29] S. Yokoyama, Scattering Amplitude and Bosonization Duality in General Chern-Simons Vector Models, JHEP 09 (2016) 105 [arXiv: 1604.01897] [INSPIRE].

[30] K. Inbasekar, S. Jain, P. Nayak and V. Umesh, All tree level scattering amplitudes in Chern-Simons theories with fundamental matter, Phys. Rev. Lett. 121 (2018) 161601 [arXiv: 1710.04227] [INSPIRE].

[31] K. Inbasekar et al., Dual Superconformal Symmetry of $\mathcal{N}=2$ Chern-Simons theory with Fundamental Matter and Non-Renormalization at Large N, arXiv:1711.02672 [INSPIRE].

[32] F. Benini, C. Closset and S. Cremonesi, Comments on 3d Seiberg-like dualities, JHEP 10 (2011) 075 [arXiv:1108.5373] [INSPIRE].

[33] O. Aharony, G. Gur-Ari and R. Yacoby, $d=3$ Bosonic Vector Models Coupled to Chern-Simons Gauge Theories, JHEP 03 (2012) 037 [arXiv:1110.4382] [INSPIRE].

[34] W.A. Bardeen and M. Moshe, Spontaneous breaking of scale invariance in a $D=3 \mathrm{U}(N)$ model with Chern-Simons gauge fields, JHEP 06 (2014) 113 [arXiv:1402.4196] [INSPIRE].

[35] W.A. Bardeen, The Massive Fermion Phase for the $\mathrm{U}(N)$ Chern-Simons Gauge Theory in $D=3$ at Large N, JHEP 10 (2014) 39 [arXiv:1404.7477] [INSPIRE]. 
[36] V. Gurucharan and S. Prakash, Anomalous dimensions in non-supersymmetric bifundamental Chern-Simons theories, JHEP 09 (2014) 009 [Erratum ibid. 1711 (2017) 045] [arXiv: 1404.7849] [INSPIRE].

[37] Y. Frishman and J. Sonnenschein, Large N Chern-Simons with massive fundamental fermions - A model with no bound states, JHEP 12 (2014) 165 [arXiv:1409.6083] [INSPIRE].

[38] M. Moshe and J. Zinn-Justin, 3D Field Theories with Chern-Simons Term for Large $N$ in the Weyl Gauge, JHEP 01 (2015) 054 [arXiv:1410.0558] [InSPIRE].

[39] G. Gur-Ari, S.A. Hartnoll and R. Mahajan, Transport in Chern-Simons-Matter Theories, JHEP 07 (2016) 090 [arXiv: 1605.01122] [INSPIRE].

[40] S. Giombi, Higher Spin - CFT Duality, in Proceedings, Theoretical Advanced Study Institute in Elementary Particle Physics: New Frontiers in Fields and Strings (TASI 2015), Boulder U.S.A. (2015), pg. 137 [arXiv:1607.02967] [INSPIRE].

[41] S.R. Wadia, Chern-Simons theories with fundamental matter: A brief review of large $N$ results including Fermi-Bose duality and the S-matrix, Int. J. Mod. Phys. A 31 (2016) 1630052.

[42] S. Giombi, V. Gurucharan, V. Kirilin, S. Prakash and E. Skvortsov, On the Higher-Spin Spectrum in Large N Chern-Simons Vector Models, JHEP 01 (2017) 058 [arXiv: 1610.08472] [INSPIRE].

[43] T. Nosaka and S. Yokoyama, Complete factorization in minimal $\mathcal{N}=4$ Chern-Simons-matter theory, JHEP 01 (2018) 001 [arXiv:1706.07234] [INSPIRE].

[44] S. Giombi, Testing the Boson/Fermion Duality on the Three-Sphere, arXiv:1707.06604 [INSPIRE].

[45] V. Guru Charan and S. Prakash, On the Higher Spin Spectrum of Chern-Simons Theory coupled to Fermions in the Large Flavour Limit, JHEP 02 (2018) 094 [arXiv:1711.11300] [INSPIRE].

[46] O. Aharony, S. Jain and S. Minwalla, to appear.

[47] O. Aharony, P. Narayan and T. Sharma, On monopole operators in supersymmetric Chern-Simons-matter theories, JHEP 05 (2015) 117 [arXiv: 1502.00945] [INSPIRE].

[48] D. Radičević, Disorder Operators in Chern-Simons-Fermion Theories, JHEP 03 (2016) 131 [arXiv: 1511.01902] [INSPIRE].

[49] P.-S. Hsin and N. Seiberg, Level/rank Duality and Chern-Simons-Matter Theories, JHEP 09 (2016) 095 [arXiv : 1607.07457] [INSPIRE].

[50] D. Radičević, D. Tong and C. Turner, Non-Abelian 3d Bosonization and Quantum Hall States, JHEP 12 (2016) 067 [arXiv: 1608.04732] [INSPIRE].

[51] A. Karch, B. Robinson and D. Tong, More Abelian Dualities in $2+1$ Dimensions, JHEP 01 (2017) 017 [arXiv: 1609.04012] [INSPIRE].

[52] O. Aharony, F. Benini, P.-S. Hsin and N. Seiberg, Chern-Simons-matter dualities with SO and USp gauge groups, JHEP 02 (2017) 072 [arXiv: 1611.07874] [INSPIRE].

[53] F. Benini, P.-S. Hsin and N. Seiberg, Comments on global symmetries, anomalies and duality in $(2+1) d$, JHEP 04 (2017) 135 [arXiv: 1702.07035] [INSPIRE]. 
[54] D. Gaiotto, Z. Komargodski and N. Seiberg, Time-reversal breaking in $Q C D_{4}$, walls and dualities in $2+1$ dimensions, JHEP 01 (2018) 110 [arXiv:1708.06806] [INSPIRE].

[55] K. Jensen and A. Karch, Bosonizing three-dimensional quiver gauge theories, JHEP 11 (2017) 018 [arXiv: 1709. 01083] [INSPIRE].

[56] K. Jensen and A. Karch, Embedding three-dimensional bosonization dualities into string theory, JHEP 12 (2017) 031 [arXiv:1709. 07872] [INSPIRE].

[57] J. Gomis, Z. Komargodski and N. Seiberg, Phases Of Adjoint QCD 3 And Dualities, SciPost Phys. 5 (2018) 007 [arXiv:1710.03258] [INSPIRE].

[58] C. Cordova, P.-S. Hsin and N. Seiberg, Global Symmetries, Counterterms and Duality in Chern-Simons Matter Theories with Orthogonal Gauge Groups, SciPost Phys. 4 (2018) 021 [arXiv: 1711.10008] [INSPIRE].

[59] F. Benini, Three-dimensional dualities with bosons and fermions, JHEP 02 (2018) 068 [arXiv: 1712.00020] [INSPIRE].

[60] K. Jensen, A master bosonization duality, JHEP 01 (2018) 031 [arXiv:1712.04933] [INSPIRE].

[61] O. Aharony, J. Marsano, S. Minwalla, K. Papadodimas and M. Van Raamsdonk, The Hagedorn - deconfinement phase transition in weakly coupled large $N$ gauge theories, Adv. Theor. Math. Phys. 8 (2004) 603 [hep-th/0310285] [INSPIRE]. 